\title{
Asymptotic Orbits in a Free Fermi Gas
}

\author{
R. Haag \\ II. Institut für Theoretische Physik der Universität Hamburg \\ R. V. Kadison \\ Department of Mathematics, University of Pennsylvania \\ D. Kastler \\ Centre Universitaire de Marseille-Luminy
}

Received June 18, 1973

\begin{abstract}
We consider the time evolution of local observables and physical states in an infinite system of non-interacting Fermi particles. The orbit of an observable in the $C^{*}$-algebra of the canonical anticommutation relations is proved to be asymptotic to a set of observables consisting of sums of products of elements of grade two and lower with support in a family of separated cells in $\mathbb{R}^{3}$ (a lacunary paving of $\mathbb{R}^{3}$ ) under time evolution. A space-factorization ("clustering") property for primary, even, locally Fock states is established. A class of such states whose space-correlations decay as $(\log d)^{-(1+a)}$ with $a$ positive and $d$ the (space-) separation is, then, proved to be time-asymptotic to their associated quasi-free states.
\end{abstract}

\section{§ 1. Introduction}

Statistical mechanics results from a "coarse grained" description of a system, i.e., from the process of limiting attention to a restricted set of observàbles. It has been the hope underlying many recent studies in Quantum Statistical Mechanics that, for most purposes, it is adequate to take for this set the local observables in an infinitely ex tended medium. If this is so then we should be able to show that time evolution of states over the algebra of local observables in an infinite system is such that a considerable simplification results as the time $t$ tends to infinity (insensitivity to initial conditions): the orbits of large classes of states should coalesce (become asymptotic to each other) as $t \rightarrow \infty$. While it appears forbiddingly difficult to obtain any rigorous mathematical results about asymptotic orbits of states for systems of infinitely many interacting particles, it is possible to study this problem for the free Fermi gas (an infinite system of non-interacting particles). From the physical point of view, the free system is, of course, a poor example to demonstrate the asymptotic simplification because interparticle collisions 
(which are absent in this case) are expected to be a principal agent in bringing about the asymptotic coalescence of states. Nevertheless we find that, even for the free gas, there is a very significant asymptotic simplification.

Our goal is Theorem 5.5 in which we show that a physical state $\varrho$ whose long-range (space-)correlations decrease sufficiently rapidly to zero for large distances, is asymptotic under time evolution to its associated quasi-free state $\hat{o}$ (the quasi-free state with the same two-point function). The main tool in the proof of this theorem is Theorem 2.3 which describes the orbit of an observables as being asymptotic to the observables from a special set. Each of the observables in this set is a (norm-limit of a) sum of products of observables of grade two or lower with support in a "cell" of a certain ("lacunary") "paving" of $\mathbb{R}^{3}$. In computing the expectation value of such an observable in a state $\varrho$ whose long range correlations decrease rapidly, we may effectively replace $\varrho$ by its "factorization" $\varrho^{\times}$relative to the paving. For the observables in question, the expectation values in $\varrho^{\times}$are precisely equal to those in $\hat{\varrho}^{\times}$(the factorization of the associated quasi-free state) and these, in turn, are effectively equal to those in $\hat{\varrho}$.

In loose outline, the preceding remarks describe the structure of the argument proving Theorem 5.5. With the exception of $\S 4$, the following sections provide the precise mathematical proof of this theorem. In $\S 4$ we prove that each even, primary, locally Fock state has long-range correlations which decay with increasing space-separation. This result lends force to the study of the class of states having prescribed rates of decay in space-correlations (carried out in $\S 5$ ). In $\S 3$ we develop the apparatus of factorization of even states essential to the application of Theorem 2.3 to the study of the time-asymptotic behavior of states. One form of this factorization is developed in $[5 ; \S 5]$. In addition, we must develop a "commutative" factorization and relate it to the "anti-commutative" factorization of $[5 ; \S 5]$. (We are deeply grateful to Robert Powers for valuable discussions of [5] and other topics involved in the final phase of this work.)

Parts of this research have been discussed in $[1,2]$. At the point where we had established the asymptotic form of the orbits of observables, Lanford and Robinson [4] were able to prove our conjecture of 'asymptotic equivalence with quasi-free states' for the class of translationally-invariant states with square-integrable truncated $n$-point functions.

For the most part, the notation and conventions we use will be explained as needed (section by section). When the CAR algebra, the $C^{*}$-algebra of the canonical anticommutation relations, appears, it will be denoted by $\mathfrak{A}$. The annihilators and creators with wave function $f$ 
(in $L_{2}\left(\mathbb{R}^{3}\right)$ ) are denoted by $a(f)$ and $a^{*}(f)$, respectively; and $a^{\prime}(f)$ is used to designate either this annihilator or creator (when it is worthwhile, notationally, to leave the specification ambiguous). Following usual notation, we write $\mathfrak{A}^{\prime}$ for the commutant of a family $\mathfrak{A}$ of operators acting on a Hilbert space ( $\mathfrak{I}^{\prime}$ is the set of bounded operators on that space commuting with all operators in $\mathfrak{A}$ ). The weak-operator closure of $\mathfrak{A}$ is denoted by $\mathfrak{A}^{-}$. When we speak of the CAR algebra based on $\mathscr{H}$, we refer to the $C^{*}$-algebra generated by the annihilators $a(f)$ with $f$ in $\mathscr{H}$. We use the notation $\mathfrak{A}(M)$ to denote the $C^{*}$-subalgebra of $\mathfrak{A}$ generated by $a(f)$ with $f$ in the subspace $M$ of $\mathscr{H}$. If $\mathscr{H}$ is $L_{2}\left(\mathbb{R}^{3}\right)$ and $\mathcal{O}$ is a region in $\mathbb{R}^{3}$, we write $\mathfrak{A}(\mathcal{O})$ in place of $\mathfrak{A}(M)$, where $M$ is the subspace of $L_{2}\left(\mathbb{R}^{3}\right)$ consisting of functions vanishing (almost everywhere) outside of $\mathcal{O}$.

\section{§ 2. The Asymptotic Form of Orbits}

In this section the following notational conventions are used. We denote by $\mathscr{H}$ the Hilbert space, $L_{2}\left(\mathbb{R}^{3}\right)$, of complex-valued, squareintegrable functions on 3-dimensional Euclidean space $\mathbb{R}^{3}$. Its elements will be denoted by $f, g$, etc., the scalar product by $(f, g)$, the norm by $\|f\|_{2}\left(=(f, f)^{\frac{1}{2}}\right)$. We deal with the CAR algebra $\mathfrak{A}$ over $\mathscr{H}$ - that is, the $C^{*}$-algebra generated by a system of elements $a(f)$, conjugatelinear in $f$ (in $\mathscr{H}$ ) and satisfying the canonical anticommutation relations (CAR):

$$
[a(f), a(g)]_{+}=\left[a^{*}(f), a^{*}(g)\right]_{+}=0, \quad\left[a^{*}(f), a(g)\right]_{+}=(f, g)
$$

where $f, g$ are in $\mathscr{H}, a^{*}(f)$ is the adjoint of $a(f)$ and $[A, B]_{+}=A B+B A$. Recall that $\|a(f)\|=\left\|a^{*}(f)\right\|=\|f\|_{2}$, where $\|A\|$ is the bound of the operator $A$ in $\mathfrak{A}$. With $x\left(=\left(x_{1}, x_{2}, x_{3}\right)\right)$ and $x^{\prime}\left(=\left(x_{1}^{\prime}, x_{2}^{\prime}, x_{3}^{\prime}\right)\right)$ in $\mathbb{R}^{3}$, $\|x\|=\left(x_{1}^{2}+x_{2}^{2}+x_{3}^{2}\right)^{\frac{1}{2}}$ and $\left(x, x^{\prime}\right)=x_{1} x_{1}^{\prime}+x_{2} x_{2}^{\prime}+x_{3} x_{3}^{\prime}$.

With $f$ in $\mathscr{H}$, we denote by $f_{(t)}$ the element $\left(\exp \left(-\mathrm{i} H_{0} t\right)\right) f$ of $\mathscr{H}$ where $H_{0}=-\frac{1}{2} \Delta$, and by $\alpha_{t}(a(f))$ the element $a\left(f_{(t)}\right)$ of $\mathfrak{U}$. In this notation, $f_{(t)}$ is the solution of the free Schrödinger equation with initial condition, $f_{(0)}=f$; and $t \rightarrow \alpha_{t}$ extends to the one-parameter automorphism group of time translations of $\mathfrak{A}$ specified by its action on the generators $a(f)$ of $\mathfrak{Q}$.

We use the notation $B_{R}$ to refer to the closed ball with center 0 and radius $R$ in $\mathbb{R}^{3}$. The term "essentially" is used in the technical measure-theoretic sense; so that two essentially disjoints subsets of $\mathbb{R}^{3}$ are subsets whose intersection has (Lebesgue) measure 0 ; and an essentially bounded set is one which is essentially disjoint from the complement of some $B_{R}$. The essential diameter of a subset $\mathscr{S}$ of $\mathbb{R}^{3}$ is the infimum of the diameter of those closed balls which essentially contain $\mathscr{S}$. 
The essential outer distance of $\mathscr{S}$ from $0 \operatorname{is} \inf \{R: \mathscr{S}$ is essentially contained in $\left.B_{R}\right\}(=R(\mathscr{S}))$. We denote by $\mathscr{S}^{\prime}$ the complement in $\mathbb{R}^{3}$ of the subset $\mathscr{S}$.

2.1. Definition. A lacunary paving $\mathscr{P}$ of $\mathbb{R}^{3}$ is a denumerable collection $\left\{\mathscr{U}_{n}\right\}$ of essentially bounded, mutually essentially disjoint subsets of $\mathbb{R}^{3}$ satisfying:

a) $\alpha(R) \rightarrow 0$ as $R \rightarrow \infty$, where $\alpha(R)$ is the quotient of the measure of $B_{R} \cap \mathscr{U}^{\prime}$ by that of $B_{R}$ and $\mathscr{U}=\bigcup_{n} \mathscr{U}_{n}$;

b) $r(R) R^{-1} \rightarrow 0$ as $R \rightarrow \infty$, where $r(R)=\sup \left\{r_{n}: R\left(\mathscr{U}_{n}\right) \leqq R\right\}$ and $r_{n}$ is the essential diameter of $\mathscr{U}_{n}$.

Concerning these pavings, a) imposes the condition that "asymptotically, they fill almost all of space"; and b) imposes the condition that, while the diameters of the $\mathscr{U}_{n}$ may grow large, the diameters of distant $\mathscr{U}_{n}$ are small by comparison with their (essential outer) distances from the origin. We note that the collection $\mathscr{P}_{0}$ of $\mathscr{U}_{n}$ in $\mathscr{P}$ lying (essentially) outside a given essentially bounded subset of $\mathbb{R}^{3}$ constitutes, again, a lacunary paving; for $r_{0}(R) \leqq r(R)$ and $\alpha_{0}(R) \leqq \alpha(R)+V_{0}\left(\frac{4}{3} \pi R^{3}\right)^{-1}$, where $\alpha_{0}$ and $r_{0}$ are the functions of a) and b) for $\mathscr{P}_{0}$, and $V_{0}$ is the measure of the bounded subset of $\mathbb{R}^{3}$ "excised" from the paving $\mathscr{P}$. We note, too, that a translate $\mathscr{P}_{0}\left(=\left\{\mathscr{U}_{n}-x_{0}\right\}\right)$ of $\mathscr{P}$ is a lacunary paving. In this case, $B_{R} \cap\left(\mathscr{U}-x_{0}\right)^{\prime}+x_{0} \cong B_{R+\left\|x_{0}\right\|} \cap \mathscr{U}^{\prime}$; so that:

$$
\alpha_{0}(R) \leqq \alpha\left(R+\left\|x_{0}\right\|\right)\left(R+\left\|x_{0}\right\|\right)^{3} R^{-3} \rightarrow 0 \text { as } R \rightarrow \infty .
$$

In addition, $R\left(\mathscr{U}_{n}\right) \leqq R+\left\|x_{0}\right\|$ if $R\left(\mathscr{U}_{n}-x_{0}\right) \leqq R$; so that:

and

$$
r_{0}(R) \leqq r\left(R+\left\|x_{0}\right\|\right)
$$

$$
r_{0}(R) R^{-1} \leqq r\left(R+\left\|x_{0}\right\|\right)\left(R+\left\|x_{0}\right\|\right)^{-1}\left(R+\left\|x_{0}\right\|\right) R^{-1} \rightarrow 0,
$$

as $R \rightarrow \infty$.

The interiors of the cubes formed by the lattice of points outside some $B_{R}$ with integer coordinates is an example of a lacunary paving.

2.2. Lemma. If $\mathscr{P}\left(=\left\{\mathscr{U}_{n}\right\}\right)$ is a lacunary paving of $\mathbb{R}^{3}$, then for each $f$ in $L_{2}\left(\mathbb{R}^{3}\right)$ and each $n$ there is a function $h_{n}$ (of $\left.t\right)$ such that $\left\|f_{(t)}-g_{t}\right\|_{2} \rightarrow 0$ as $t \rightarrow \infty$, where $g_{t}(x)=h_{n}(t) \exp \left[\frac{1}{2} i t^{-1}\|x\|^{2}\right]$ for $x$ in $\mathscr{U}_{n}$ and $g_{t}$ vanishes on $\mathscr{U}^{\prime}$.

2.3. Theorem. If $\mathfrak{A}_{t, n}$ is the 4-dimensional subalgebra of $\mathfrak{A}$ generated by $I, a\left(g_{t, n}\right)$ and $a^{*}\left(g_{t, n}\right)$, where $g_{t, n}(x)=h_{n}(t) \exp \left[\frac{1}{2} i t^{-1}\|x\|^{2}\right]$ for $x$ in $\mathscr{U}_{n}$ and $g_{t, n}$ vanishes on $\mathscr{U}_{n}^{\prime}$, and $\mathfrak{I}_{t}^{\mathscr{P}}$ is the $C^{*}$-subalgebra of $\mathfrak{A}$ generated by $\left\{\mathfrak{U}_{t, n}\right\}$, then, for each $A$ in $\mathfrak{A}$, there is a one-parameter family $\left(B_{t}\right)$, with $B_{t}$ in $\mathfrak{O}_{t}^{\mathscr{P}}$, such that $\left\|\alpha_{t}(A)-B_{t}\right\| \rightarrow 0$ as $t \rightarrow \infty$ (that is, $\alpha_{t}(A)$ is asymptotic in time to $\mathfrak{P}_{t}^{\mathscr{P}}$ ). 
Before beginning the proofs, we remark that the heuristic basis for the lemma resides in two properties of the solutions of the free Schrödinger equation at large times. The first is that the wave function $f_{(t)}(x)$ spreads over a very large region of space, the diameter of which increases proportionally to $t$. Thus, for a fixed lacunary paving, as a result of a), the $L_{2}$-norm of the part of the wave function $f_{(t)}$ which has support in the "unpaved" part of space will tend to 0 as $t \rightarrow \infty$. The second is, that independently of the initial shape of the wave function, the shape of $f_{(t)}$, within a small region of space around a point $x_{n}$, is, in essence, that of a plane wave with wave vector $t^{-1} x_{n}$, at large times $t$. A slightly better approximation, one which turns out to be valid to a sufficient degree of accuracy within each $\mathscr{U}_{n}$ when the size of the $\mathscr{U}_{n}$ is limited by b) is given by $h_{n}(t) g_{t, n}(x)$.

Assuming, for the moment, that the lemma has been established, we proceed to the

Proof of Theorem 2.3. Note that

$$
\left\|\alpha_{t}(a(f))-a\left(g_{t}\right)\right\|=\left\|a\left(f_{(t)}\right)-a\left(g_{t}\right)\right\|=\left\|a\left(f_{(t)}-g_{t}\right)\right\|=\left\|f_{(t)}-g_{t}\right\|_{2}
$$

so that $\| \alpha_{t}\left(a(f)-a\left(g_{t}\right) \| \rightarrow 0\right.$ as $t \rightarrow 0$, assuming Lemma 2.2. As $\sum_{n} g_{t, n}$ converges to $g_{t}$ in $\mathscr{H}, \sum_{n} a\left(g_{t, n}\right)$ converges to $a\left(g_{t}\right)$ in the norm topology on bounded operators; whence $a\left(g_{t}\right)$ lies in $\mathfrak{A}_{t}^{\mathscr{P}}$ (in fact, $a\left(g_{t}\right)$ is in the norm closure of the linear span of the $\left.\mathfrak{U}_{t, n}\right)$. Taking $a\left(g_{t}\right)$ as $B_{t}$, our theorem is established for the generators $a(f)$ of $\mathfrak{A}$. Since $\left\|\alpha_{t}(a(f))-B_{t}\right\|$ $=\left\|\alpha_{t}(a(f))^{*}-B_{t}^{*}\right\|=\left\|\alpha_{t}\left(a^{*}(f)\right)-B_{t}^{*}\right\|$, and $B_{t}^{*} \in \mathfrak{O}_{t}^{\mathscr{P}}$, the same is true for $a^{*}(f)$. If $p$ is a (non-commutative) polynomial in several variables (regarded as a mapping from $\mathfrak{A} \times \cdots \times \mathfrak{A}$ to $\mathfrak{A}$ ), then $p$ on bounded sets is uniformly norm-continuous, and $\alpha_{t} \circ p=p \circ \bar{\alpha}_{t}$ (where $\bar{\alpha}_{t}$ is the extension of $\alpha_{t}$ to $\mathfrak{A} \times \cdots \times \mathfrak{U}$ obtained by applying $\alpha_{t}$ to each coordinate). Since $\mathfrak{Y}_{t}^{\mathscr{P}}$ is a ${ }^{*}$-algebra, $p$ maps $\mathfrak{I}_{t}^{\mathscr{P}} \times \cdots \times \mathfrak{\mathfrak { P }}_{t}^{\mathscr{P}}$ into $\mathfrak{I}_{t}^{\mathscr{P}}$. It follows that the assertion of the theorem holds for the operators in the norm-dense, *-subalgebra $\mathfrak{A}_{0}$ of $\mathfrak{A}$ consisting of polynomials in the generators $a(f), a^{*}(f)$.

For arbitrary $A$ in $\mathfrak{A}$, choose $A_{m}$ in $\mathfrak{U}_{0}$ so that $\left\|A-A_{m}\right\| \leqq m^{-1}$. Since $A_{m} \in \mathfrak{A}_{0}$, there is a one-parameter family $\left(B_{t, m}\right)$ such that $\left\|\alpha_{t}\left(A_{m}\right)-B_{t, m}\right\| \rightarrow 0$ as $t \rightarrow \infty$, with $B_{t, m}$ in $\mathfrak{U}_{t}^{\mathscr{P}}$. Choose $t_{1}$, so that $\left\|\alpha_{t}\left(A_{1}\right)-B_{t, 1}\right\| \leqq 1$ if $t_{1} \leqq t$ and $t_{m}$, inductively, so that $t_{m-1}+1 \leqq t_{m}$ and, if $t_{m} \leqq t$ then $\left\|\alpha_{t}\left(A_{m}\right)-B_{t, m}\right\| \leqq m^{-1}$. Let $B_{t}$ be 0 for $0 \leqq t \leqq t_{1}$, and $B_{t}$ be $B_{t, m}$ for $t_{m}<t \leqq t_{m+1}$. Then $\left\|\alpha_{t}(A)-B_{t}\right\| \rightarrow 0$ as $t \rightarrow \infty$; for, if $t_{m}<t \leqq t_{m+1}$,

$$
\begin{aligned}
\left\|\alpha_{t}(A)-B_{t}\right\| & =\left\|\alpha_{t}(A)-B_{t, m}\right\| \leqq\left\|\alpha_{t}(A)-\alpha_{t}\left(A_{m}\right)\right\|+\left\|\alpha_{t}\left(A_{m}\right)-B_{t, m}\right\| \\
& =\left\|A-A_{m}\right\|+\left\|\alpha_{t}\left(A_{m}\right)-B_{t, m}\right\| \leqq 2 m^{-1} .
\end{aligned}
$$


Proof of Lemma 2.2. It will suffice to establish the assertion of the lemma for each function in a total family; for, by linearity of $f \rightarrow f_{(t)}$, the same then holds for each function in the dense linear subspace $\mathscr{H}_{0}$ consisting of finite linear combinations of functions in the total family. For an arbitrary $f$ in $\mathscr{H}$, choose $f_{m}$ in $\mathscr{H}_{0}$ such that $\left\|f-f_{m}\right\|_{2} \leqq m^{-1}$ and $\left(g_{t, m}\right)$ so that $\left\|f_{m,(t)}-g_{t, m}\right\|_{2} \rightarrow 0$ as $t \rightarrow \infty$. Arguing, now, precisely as above, we find $\left(g_{t}\right)$ such that $\left\|f_{(t)}-g_{t}\right\|_{2} \rightarrow 0$.

As our total family, we use the normalized Gaussians with various centers and dispersions. If $f(x)=\pi^{-\frac{3}{4}} s^{-\frac{3}{2}} \exp \left[-\frac{1}{2} s^{-2}\left\|x-x_{0}\right\|^{2}\right]$, then $f_{(t)}(x)=\pi^{-\frac{3}{4}} S^{\frac{3}{2}}\left(s^{2}+i t\right)^{-\frac{3}{2}} \exp \left[-\frac{1}{2}\left(s^{2}+i t\right)^{-1}\left\|x-x_{0}\right\|^{2}\right]$. Let $\tilde{g}_{t, n}(x)$ be $f_{(t)}(x)$, for $x$ in $\mathscr{U}_{n}$, and vanish on $\mathscr{U}_{n}^{\prime}$. Let $\tilde{g}_{t}(x)$ be $f_{(t)}(x)$, for $x$ in $\mathscr{U}$, and vanish on $\mathscr{U}^{\prime}$. Given a positive $\varepsilon(<1)$, choose $c$ exceeding 1 such that $4 \pi \int_{\frac{1}{2} c}^{\infty} u^{2} \exp u \exp -\frac{1}{4} u^{2} d u \leqq \frac{\varepsilon^{2}}{2}$. Then $\int_{c}^{\infty} u^{2} \exp -u^{2} d u \leqq \frac{\varepsilon^{2}}{6}$. Let $\mathscr{P}_{0}$, with associated $\mathscr{U}_{0}$ and $\alpha_{0}$, be the paving $\mathscr{P}$ translated by $-x_{0}$. Choose $R_{0}$ such that $\alpha_{0}(\bar{R})<\frac{1}{2} c^{-3} \varepsilon^{2}$ if $\bar{R} \geqq R_{0}$. Write $k(t)$ for $s\left(s^{4}+t^{2}\right)^{-\frac{1}{2}}$. Then $k(t) \rightarrow 0$ as $t \rightarrow \infty$; so that there is a positive $t_{0}$ such that $c k(t)^{-1} \geqq R_{0}$ if $t \geqq t_{0}$. Writing $R$ for $c k(t)^{-1}$, note that, if $t \geqq t_{0}$,

$$
\begin{aligned}
\left\|f_{(t)}-\tilde{g}_{t}\right\|_{2}^{2} & =\pi^{-\frac{3}{2}} k(t)^{3} \int_{\mathcal{U}^{\prime}} \exp -\left(k(t)\left\|x-x_{0}\right\|\right)^{2} d x \\
& =\pi^{-\frac{3}{2}} k(t)^{3} \int_{\mathscr{U}_{0}^{\prime}} \exp -(k(t)\|x\|)^{2} d x \\
& \leqq \pi^{-\frac{3}{2}} k(t)^{3}\left[\int_{B_{R} \cap \mathcal{U}_{0}^{\prime}} \exp -(k(t)\|x\|)^{2} d x+\int_{B_{R}^{\prime}} \exp -(k(t)\|x\|)^{2} d x\right] \\
& \leqq \pi^{-\frac{3}{2}} k(t)^{3}\left[\int_{B_{R} \cap \mathcal{U}_{0}^{\prime}} d x+4 \pi \int_{R}^{\infty} y^{2} \exp -(k(t) y)^{2} d y\right] \\
& \leqq \pi^{-\frac{3}{2}} k(t)^{3}\left[\frac{4}{3} \pi R^{3} \alpha_{0}(R)+4 \pi k(t)^{-3} \int_{k(t) R}^{\infty} u^{2} \exp -u^{2} d u\right] \\
& =4 \pi^{-\frac{1}{2}}\left[\frac{1}{3} c^{3} \alpha_{0}(R)+\int_{c}^{\infty} u^{2} \exp -u^{2} d u\right] \\
& \leqq 3\left(\frac{\varepsilon^{2}}{6}+\frac{\varepsilon^{2}}{6}\right)=\varepsilon^{2} .
\end{aligned}
$$

Thus $\left\|f_{(t)}-\tilde{g}_{t}\right\|_{2} \rightarrow 0$ as $t \rightarrow \infty$; and it remains to show that $\left\|g_{t}-\tilde{g}_{t}\right\|_{2} \rightarrow 0$ as $t \rightarrow \infty$, where $g_{t}$ is of the form described in the statement of the lemma. Toward this end, choose a point $x_{n}$ in each $\mathscr{U}_{n}$. Note that, for $x$ in $\mathscr{U}_{n}$, writing $v_{n}(t)$ for $\pi^{-\frac{3}{4}} S^{\frac{3}{2}}\left(s^{2}+i t\right)^{-\frac{3}{2}} \exp \left[-\frac{1}{2}\left(s^{2}+i t\right)^{-1}\left\|x_{n}-x_{0}\right\|^{2}\right], \tilde{g}_{t, n}(x)$ is

$$
v_{n}(t) \exp \left[\left(s^{2}+i t\right)^{-1}\left(x-x_{n}, x_{0}\right)\right] \exp \left[-\frac{1}{2}\left(s^{2}+i t\right)^{-1}\left(\|x\|^{2}-\left\|x_{n}\right\|^{2}\right)\right] .
$$


Let $g_{t, n}(x)$ be 0 on $\mathscr{U}_{n}^{\prime}$; and for $x$ in $\mathscr{U}_{n}$, let $g_{t, n}(x)$ be

$$
v_{n}(t) \exp \left[\frac{1}{2} i t^{-1}\left(\|x\|^{2}-\left\|x_{n}\right\|^{2}\right)\right] \text { (of the form } h_{n}(t) \exp \left[\frac{1}{2} i t^{-1}\|x\|^{2}\right] \text { ). }
$$

Removing from each $\mathscr{U}_{n}$ a set of measure 0 , we may assume that the essential and actual diameters $r_{n}$ of each $\mathscr{U}_{n}$ are the same, that the essential and actual outer distances $R_{n}$ of $\mathscr{U}_{n}$ from 0 coincide, that $x_{n}$ is chosen in the altered $\mathscr{U}_{n}$, that distinct $\mathscr{U}_{n}$ are disjoint, and that each $\mathscr{U}_{n}$ is bounded. We use the same symbols to designate the sets and paving as altered as well as the restrictions of $\tilde{g}_{t, n}, \tilde{g}_{t}, g_{t, n}$ and $g_{t}$ to the altered paving. Since these functions have been modified on a set of measure 0 , the $L_{2^{-}}$ estimates obtained for them apply equally to the original functions.

With the notation as before, let $R_{1}\left(>16\left\|x_{0}\right\|\right)$ be such that $r(\bar{R}) \bar{R}^{-1}$ $\leqq \varepsilon\left[4 c\left(3 c+s^{-1}\left\|x_{0}\right\|\right)\right]^{-1}$ if $2 \bar{R} \geqq R_{1}$. Since $k(t) \rightarrow 0$ and $t k(t) \rightarrow s$ as $t \rightarrow \infty$, there is a $t_{1}$, such that $2 c k(t)^{-1}(=2 R) \geqq R_{1}, 3 t k(t) \geqq s$ and $1 \geqq 6\left\|x_{0}\right\| k(t)$ if $t \geqq t_{1}$. Suppose $\mathscr{U}_{n} \leqq B_{R}, x \in \mathscr{U}_{n}$ and $t \geqq t_{1}$. Letting $q(u)$ be $u \exp u$, and using the inequality $|1-\exp u| \leqq q(|u|)$, we have:

$$
\begin{aligned}
\mid 1- & g_{t, n}(x) \tilde{g}_{t, n}(x)^{-1} \mid \\
& =\left|1-\exp \left[\frac{1}{2} i t^{-1} s^{2}\left(s^{2}+i t\right)^{-1}\left(\|x\|^{2}-\left\|x_{n}\right\|^{2}\right)-\left(s^{2}+i t\right)^{-1}\left(x-x_{n}, x_{0}\right)\right]\right| \\
& \leqq q\left(\frac{1}{2} t^{-1} s k(t)\left|\|x\|^{2}-\left\|x_{n}\right\|^{2}\right|+s^{-1} k(t)\left\|x_{0}\right\|\left\|x-x_{n}\right\|\right) \\
& \leqq q\left(s t^{-1} r_{n} R k(t)+s^{-1} k(t)\left\|x_{0}\right\| r_{n}\right) \\
& \leqq q\left(r(R) R^{-1} k(t) R\left(s t^{-1} R+s^{-1}\left\|x_{0}\right\|\right)\right) \\
& \leqq \varepsilon\left[4 c\left(3 c+s^{-1}\left\|x_{0}\right\|\right)\right]^{-1} c\left(3 c+s^{-1}\left\|x_{0}\right\|\right) \exp \frac{1}{4} \varepsilon \\
& \leqq \frac{1}{4} \varepsilon \exp \frac{1}{4} \leqq \frac{\varepsilon}{2} .
\end{aligned}
$$

Thus

$$
\begin{aligned}
\sum_{\mathcal{U}_{n} \leqq B_{R}}\left\|\tilde{g}_{t, n}-g_{t, n}\right\|_{2}^{2} & \leqq \frac{1}{4} \varepsilon^{2} \sum_{\mathscr{U} \leqq B_{R}}\left\|\tilde{g}_{t, n}\right\|_{2}^{2} \\
& \leqq \frac{1}{4} \varepsilon^{2}\left\|\tilde{g}_{t}\right\|_{2}^{2} \leqq \frac{1}{4} \varepsilon^{2}\left\|f_{(t)}\right\|_{2}^{2}=\frac{1}{4} \varepsilon^{2} .
\end{aligned}
$$

Since $\varepsilon<1$ and $1<c, \quad \varepsilon\left[4 c\left(3 c+s^{-1}\left\|x_{0}\right\|\right)\right]^{-1}<\frac{1}{12}$. If $\mathscr{U}_{m} \ddagger B_{R}$, then $R_{m} \geqq R \geqq \frac{1}{2} R_{1}$; so that $r_{m} R_{m}^{-1} \leqq r\left(R_{m}\right) R_{m}^{-1}<\frac{1}{12}$. Thus $\frac{11}{12} R_{m}<R_{m}$ $-r_{m}$; and $\frac{11}{12}\left\|x^{\prime}\right\| \leqq \frac{11}{12} R_{m}<R_{m}-r_{m} \leqq\|x\|$, for all $x$ and $x^{\prime}$ in $\mathscr{U}_{m}$. As $R_{1}>16\left\|x_{0}\right\|, R_{m}>8\left\|x_{0}\right\| ;$ and

$$
\begin{aligned}
\left\|x_{m}-x_{0}\right\| & \geqq\left\|x_{m}\right\|-\left\|x_{0}\right\| \geqq\left\|x_{m}\right\|-\frac{1}{8} R_{m} \geqq\left\|x_{m}\right\|-\frac{1}{7}\left\|x_{m}\right\| \\
& =\frac{6}{7}\left\|x_{m}\right\| \geqq \frac{6}{7}\left(\frac{7}{8}\|x\|\right)=\frac{3}{4}\|x\| .
\end{aligned}
$$


With $x$ in $\mathscr{U}_{m},\left|\tilde{g}_{t, m}(x)-g_{t, m}(x)\right|^{2}$ is

$$
\begin{aligned}
\left|v_{m}(t)\right|^{2} & \left|\exp \frac{\left(x-x_{m}, x_{0}\right)}{s^{2}+i t} \exp -\frac{\left(\|x\|^{2}-\left\|x_{m}\right\|^{2}\right)}{2\left(s^{2}+i t\right)}-\exp \frac{-\left(\|x\|^{2}-\left\|x_{m}\right\|^{2}\right)}{2 i t}\right|^{2} \\
\leqq & \left|v_{m}(t)\right|^{2}\left|1+\exp \left[\left(x-x_{m}, x_{0}\right) k(t)^{2}\right] \exp \left[-\frac{1}{2}\left(\|x\|^{2}-\left\|x_{m}\right\|^{2}\right) k(t)^{2}\right]\right|^{2} \\
\leqq & \pi^{-\frac{3}{2}} k(t)^{3} \exp \left[-\left\|x_{m}-x_{0}\right\|^{2} k(t)^{2}\right] \\
& \cdot\left|1+\exp \left[\left(x-x_{m}, x_{0}\right) k(t)^{2}\right] \exp \left[\frac{1}{2}\left(R_{m}^{2}-\left(R_{m}-r_{m}\right)^{2}\right) k(t)^{2}\right]\right|^{2} \\
\leqq & 4 \pi^{-\frac{3}{2}} k(t)^{3} \exp \left[-\frac{1}{2}\|x\|^{2} k(t)^{2}\right] \exp \left[2\left\|x-x_{m}\right\| \cdot\left\|x_{0}\right\| k(t)^{2}\right] \\
& \cdot \exp \left[2 r_{m} R_{m} k(t)^{2}\right] \\
\leqq & k(t)^{3} \exp \left[-\frac{1}{2}\|x\|^{2} k(t)^{2}\right] \exp \left[2(\|x\|+2\|x\|)\left\|x_{0}\right\| k(t)^{2}\right] \\
& \cdot \exp \left[\frac{1}{6} R_{m}^{2} k(t)^{2}\right] \\
\leqq & k(t)^{3} \exp \left[-\frac{1}{2}\|x\|^{2} k(t)^{2}\right] \exp [\|x\| k(t)] \exp \left[\frac{1}{4}(\|x\| k(t))^{2}\right] .
\end{aligned}
$$

Thus

$$
\begin{aligned}
& \sum_{\mathscr{U}_{m \neq B_{R}}\left\|\tilde{g}_{t, m}-g_{t, m}\right\|_{2}^{2}} \leqq \sum_{\mathcal{U}_{m \ddagger B_{R}}} k(t)^{3} \int_{\mathscr{U}_{m}} \exp \left[-\frac{1}{4}\|x\|^{2} k(t)^{2}\right] \exp [\|x\| k(t)] d x \\
& \leqq k(t)^{3} \int_{B_{\frac{1}{2} R}} \exp \left[-\frac{1}{4}\|x\|^{2} k(t)^{2}\right] \exp [\|x\| k(t)] d x \\
&=4 \pi k(t)^{3} \int_{\frac{1}{2} R}^{\infty} y^{2} \exp -\frac{1}{4}(y k(t))^{2} \exp [y k(t)] d y \\
&=4 \pi \int_{\frac{1}{2} R k(t)}^{\infty} u^{2} \exp -\frac{1}{4} u^{2} \exp u d u \\
&=4 \pi \int_{\frac{1}{2} c}^{\infty} u^{2} \exp u \exp -\frac{1}{4} u^{2} d u \leqq \frac{\varepsilon^{2}}{2}
\end{aligned}
$$

Collecting estimates, we have:

$$
\left\|\tilde{g}_{t}-g_{t}\right\|_{2}^{2}=\sum_{\mathcal{U}_{n} \leqq B_{R}}\left\|\tilde{g}_{t, n}-g_{t, n}\right\|_{2}^{2}+\sum_{\mathcal{U}_{m} \neq B_{R}}\left\|\tilde{g}_{t, m}-g_{t, m}\right\|_{2}^{2} \leqq \frac{\varepsilon^{2}}{4}+\frac{\varepsilon^{2}}{2}<\varepsilon^{2},
$$

if $t \geqq t_{1}$. Thus $\left\|f_{(t)}-g_{t}\right\|_{2} \rightarrow 0$ as $t \rightarrow \infty$, completing the proof.

\section{$\S$ 3. Factorizations of States}

With $\mathfrak{U}$ the CAR algebra based on $\mathscr{H}\left(=L_{2}\left(\mathbb{R}^{3}\right)\right)$, orthogonal decompositions of $\mathscr{H}$ give rise to (mutually) "anticommuting" subalgebras of $\mathfrak{U}$ which generate $\mathfrak{A}$. Powers shows [5; Theorem 5.4] that a family of even states, one defined on each of these subalgebras, gives 
rise to an even state of $\mathfrak{A}$ which is their "product". This process may be applied to "decouple" even states "along" an orthogonal decomposition of $\mathscr{H}$. The state is restricted to the corresponding subalgebras and the product of these restrictions is formed. If the decomposition of $\mathscr{H}$ arises from a partition of $\mathbb{R}^{3}$, then this "factorization" of the state amounts to eliminating its correlations between distinct regions of the partition.

We say that a state $\varrho$ of $\mathfrak{A}$ is "locally Fock" if the restriction of $\varrho$ to $\mathfrak{A}(M)$ is normal whenever $M$ arises from a bounded region of $\mathbb{R}^{3}$, where $\mathfrak{A}$ appears in its Fock representation and the weak-operator closure $\mathfrak{A}(M)^{-}$of $\mathfrak{A}(M)$ is taken relative to this representation. For such a subspace $M, \mathfrak{A}(M)^{-}$is a type $I_{\infty}$ factor. For a locally Fock state $\varrho$, another process is available for forming products along a decomposition of $\mathscr{H}$ arising from bounded regions of $\mathbb{R}^{3}$. Applying the GNS construction to $\varrho$, we may suppose that $\varrho$ is a vector state $\omega_{x} \mid \mathfrak{A}$. We will note (Lemma 3.2) that $\mathfrak{A}(M)^{-}$is a type $I$ factor, again, in this representation ( $M$ associated with a bounded region of $\mathbb{R}^{3}$ ). Using the simplest properties of tensor products, we can form the product of the restrictions of $\omega_{x}$ to the type $I$ factor $\mathfrak{A}(M)^{-}$and its commutant $\mathfrak{A}(M)^{\prime}$. The restriction $\varrho_{M}^{\otimes}$ of this product to $\mathfrak{A}$ is the second factorization. Denoting by $\varrho_{M}^{\times}$ the product according to Powers, we will see (Lemma 3.4) that $\varrho_{M}^{\otimes}$ and $\varrho_{M}^{\times}$coincide. For later application, this identification is needed, as well as the determination of the commutant of $\mathfrak{A}(M)$ in $\mathfrak{A}^{-}$preliminary to it.

If $\mathscr{P}$ is a collection of regions mutually disjoint in $\mathbb{R}^{3}(\mathscr{P}$ is to become a lacunary paving, at a later stage), and $\varrho$ is an even state of $\mathfrak{A}$, we denote by $\varrho_{\mathscr{P}}^{\times}$the (Powers) factorization of $\varrho$ along the decomposition of $L_{2}\left(\mathbb{R}^{3}\right)$ obtained from the regions in $\mathscr{P}$ and the complement of their union. The next five lemmas will establish the existence of the "commutant" product $\varrho_{\mathscr{P}}^{\otimes}$ for even locally Fock states $\varrho$.

3.1. Lemma. If $\left\{\mathscr{N}_{a}\right\}$ is a family of mutually commuting type I factors acting on a Hilbert space $\mathscr{H}$ and $\varrho_{a}$ is a normal state of $\mathcal{N}_{a}$, there is a unique state $\varrho$ of the $C^{*}$-algebra $\mathfrak{A}$ generated by $\left\{\mathscr{N}_{a}\right\}$ (von Neumann algebra, if $\left\{\mathscr{N}_{a}\right\}$ is a finite family) such that $\varrho\left(A_{1} \ldots A_{n}\right)=\varrho_{a_{1}}\left(A_{1}\right) \ldots \varrho_{a_{n}}\left(A_{n}\right)$, when $A_{j}$ is in $\mathscr{N}_{a_{j}}$ and $a_{1}, \ldots, a_{n}$ are distinct.

Proof. Let $f$ be the finite set $\left\{a_{1}, \ldots, a_{n}\right\}$ and $\mathfrak{Q}_{f}$ be the $C^{*}$-subalgebra of $\mathfrak{A}$ generated by $\mathscr{N}_{a_{1}}, \ldots, \mathscr{N}_{a_{n}}$. Represent $\mathscr{N}_{a_{1}}, \ldots, \mathscr{N}_{a_{n}}$ on Hilbert spaces $\mathscr{H}_{1}, \ldots, \mathscr{H}_{n}$, respectively, so that each is a type I factor with separating vector. (This can be effected by taking repeated copies of $\mathscr{N}_{a_{j}}$, if necessary.) In this representation, $\varrho_{a_{j}}=\omega_{x_{j}} \mid \mathscr{N}_{a_{j}}$ for some unit vector $x_{j}$ in $\mathscr{H}_{j}$. Form $\mathscr{H}_{1} \otimes \cdots \otimes \mathscr{H}_{n}$. Then $\mathscr{N}_{a_{1}} \otimes \cdots \otimes \mathscr{N}_{a_{n}}$ is isomorphic to the von Neumann algebra generated by $\mathscr{N}_{a_{1}}, \ldots, \mathscr{N}_{a_{n}} ;$ and $\omega_{x_{1} \otimes \cdots \otimes x_{n}}$ 
restricts to the (unique) normal state $\varrho_{f}$ of $\mathfrak{H}_{f}$ such that $\varrho_{f}\left(A_{1} \ldots A_{n}\right)$ $=\varrho_{a_{1}}\left(A_{1}\right) \ldots \varrho_{a_{n}}\left(A_{n}\right)$. (Uniqueness results from the fact that such products are a total family for this $C^{*}$-algebra.) If $f_{1} \cong f_{2}$, this uniqueness implies that $\varrho_{f_{2}}$ is an extension of $\varrho_{f_{1}}$. Thus the $\varrho_{f}$ define a bounded linear functional on the norm-dense subalgebra ${ }^{1} \vee \mathfrak{U}_{f}$ of $\mathfrak{A}$. Its unique extension to $\mathfrak{A}$ is a state $\varrho$ extending each $\varrho_{f}$. Thus $\varrho\left(A_{1} \cdots A_{n}\right)=\varrho_{a_{1}}\left(A_{1}\right) \ldots \varrho_{a_{n}}\left(A_{n}\right)$, when $A_{j}$ is in $\mathscr{N}_{a_{j}}$.

3.2. Lemma. If $\mathfrak{A}$ is the $C A R$ algebra based on $L_{2}\left(\mathbb{R}^{3}\right), \mathfrak{A}$ acting on $\tilde{\mathscr{H}}$ is the Fock representation of $\mathfrak{A}, \varrho$ is a locally Fock state of $\mathfrak{A}$, and $\pi$ is the representation of $\mathfrak{A}$ engendered by $\varrho$; then there is a (unique) state $\varrho$ of the norm closure $\mathfrak{\mathfrak { A }}$ of the union of the weak-operator closures $\mathfrak{A}(M)^{-}$ (relative to $\tilde{\mathscr{H}}$ ) of those subalgebras of $\mathfrak{A}$ corresponding to bounded regions of $\mathbb{R}^{3}$, extending @ and normal on each such $\mathfrak{A}(M)^{-}$. The representation $\tilde{\pi}$ of $\tilde{\mathfrak{A}}$ engendered by $\varrho$ restricts, on $\mathfrak{A}$, to (a representation unitarily equivalent to) $\pi$; and $\pi(\mathfrak{A}(M))^{-}$is a type I factor, when $M$ corresponds to a bounded region of $\mathbb{R}^{3}$.

Proof. Let $\mathscr{H}_{0}$ be the representation space of $\tilde{\pi}$ and $x_{0}$ be a unit vector in $\mathscr{H}_{0}$ cyclic under $\tilde{\pi}(\tilde{\mathfrak{I}})$ such that $\tilde{\varrho}(\tilde{A})=\left\langle\tilde{\pi}(\tilde{A}) x_{0} \mid x_{0}\right\rangle$ for each $\tilde{A}$ in $\tilde{\mathfrak{U}}$. Then, with $A$ in $\mathfrak{A}, \varrho(A)=\tilde{\varrho}(A)=\left\langle\tilde{\pi}(A) x_{0} \mid x_{0}\right\rangle$. Thus $\tilde{\pi} \mid \mathfrak{A}$ composed with restriction to $\left[\tilde{\pi}(\mathfrak{R}) x_{0}\right]$ is unitarily equivalent to $\pi$. We complete the proof by showing that $\left[\tilde{\pi}(\mathfrak{l}) x_{0}\right]=\mathscr{H}_{0}$.

Since $\tilde{\varrho}$ is locally Fock, $\tilde{\pi} \mid \mathfrak{A}(M)^{-}$is normal [3; proof of Proposition 8] for each $M$ associated with a bounded region of $\mathbb{R}^{3}$. Thus $\tilde{\pi}(\mathfrak{Q}(M))$ is strong-operator dense in $\tilde{\pi}\left(\mathfrak{A}(M)^{-}\right)$. With unions extended over all such $M$, it follows that

$$
\left[\tilde{\pi}(\mathfrak{H}) x_{0}\right]=\left[\vee \tilde{\pi}(\mathfrak{A}(M)) x_{0}\right]=\left[\vee \tilde{\pi}\left(\mathfrak{A}(M)^{-}\right) x_{0}\right]=\left[\tilde{\pi}(\tilde{\mathfrak{l}}) x_{0}\right]=\mathscr{H}_{0} .
$$

It follows, too, that $\pi(\mathfrak{A}(M))^{-}=\tilde{\pi}\left(\mathfrak{A}(M)^{-}\right)$is a type $I$ factor.

3.3. Lemma. If $\mathfrak{A}$ is the $C A R$ algebra based on $\mathscr{H}, M$ is a closed subspace of $\mathscr{H},\left\{f_{j}\right\}$ is an orthonormal basis for $M,\left\{g_{j}\right\}$ is an orthonormal basis for $\mathscr{H} \ominus M, \mathfrak{A}$ acts on $\mathscr{H}_{0}$ in such a way that $\mathfrak{U}(M)^{-}$is a type $\mathrm{I}$ factor and some vector state $\omega_{x}$ restricts to an even state of $\mathfrak{A}(M)$, then there is a unitary operator $U$ in $\mathfrak{A}(M)^{-}$such that $U^{2}=I, U a(f) U=-a(f)$ for $f$ in $M, U a(g) U=a(g)$ for $g$ in $\mathscr{H} \ominus M$, and $\mathfrak{A}(M)^{\prime} \wedge \mathfrak{U}^{-}$is the weakoperator closure of finite linear combinations of elements of $\mathfrak{A}^{-}$of the form $a^{\prime}\left(g_{j_{1}}\right) \ldots a^{\prime}\left(g_{j_{2 n}}\right)$ and $U a^{\prime}\left(g_{k_{1}}\right) \ldots a^{\prime}\left(g_{k_{2 m+1}}\right)$.

Proof. Let $E$ be the projection in $\mathfrak{A}(M)^{\prime}$ with range $[\mathfrak{U}(M) x]$. Since $\mathfrak{A}(M)^{-}$is a factor, $A \rightarrow A E$ is an isomorphism of $\mathfrak{A}(M)^{-}$onto $\mathfrak{Q}(M)^{-} E$. The unitary operator $f \rightarrow-f$ on $M$ gives rise to an automorphism $\alpha_{M}$ of $\mathfrak{A}(M)$. Since $\omega_{x} \mid \mathfrak{A}(M)$ is even, $\omega_{x} \circ \alpha_{M}=\omega_{x} \mid \mathfrak{A}(M)$. Thus $A x \rightarrow \alpha_{M}(A) x$ extends to a unitary operator on $[\mathfrak{U}(M) x]$ which implements the mapping

\footnotetext{
${ }^{1}$ We use $\vee$ and $\wedge$ as well as $\cup$ and $\cap$ for unions and intersections.
} 
$A E \rightarrow \alpha_{M}(A) E$ of $\mathfrak{A}(M) E$ onto itself. Hence this mapping and $\alpha_{M}$ extend to automorphisms of $\mathfrak{A}(M)^{-} E$ and $\mathfrak{A}(M)^{-}$, respectively. Letting " $\alpha_{M}$ " denote, again, the extension to $\mathfrak{A}(M)^{-}$of $\alpha_{M}$, there is a unitary operator $U$ in $\mathfrak{A}(M)^{-}$which implements $\alpha_{M}$ (since $\mathfrak{A}(M)^{-}$is a factor of type I). Since $\alpha_{M}^{2}(A)=A, U^{2}$ is a scalar; and we may assume that $U^{2}=I$.

Since $U$ is in $\mathfrak{A}(M)^{-}, U$ is the strong-operator limit of a net $\left\{T_{a}\right\}$ in (the unit ball of) $\mathfrak{A}(M)$. Thus $U$ is the strong-operator limit of the net $\left\{\frac{1}{2}\left(T_{a}+U T_{a} U\right)\right\}$ of even elements of $\mathfrak{A}(M)$. As each even element of $\mathfrak{A}(M)$ commutes with $\mathfrak{A}(\mathscr{H} \ominus M), U$ commutes with $\mathfrak{U}(\mathscr{H} \ominus M)$. Hence $U$ implements the automorphism of $\mathfrak{A}$ induced by the unitary operator $f \rightarrow-f, g \rightarrow g$ on $\mathscr{H}$, with $f$ in $M$ and $g$ in $\mathscr{H} \ominus M$. If $\mathscr{N}$ is the weak-operator closure of the algebra of finite linear combinations of elements of the form $a^{\prime}\left(g_{j_{1}}\right) \ldots a^{\prime}\left(g_{j_{2 n}}\right)$ and $U a^{\prime}\left(g_{j_{1}}\right) \ldots a^{\prime}\left(g_{j_{2 m+1}}\right)$, then each $a^{\prime}(f), f$ in $M$, commutes with $\mathscr{N}$; and $\mathscr{N} \subseteq \mathfrak{A}(M)^{\prime} \wedge \mathfrak{A}^{-}$. Moreover $\mathscr{N}$ and $\mathfrak{A}(M)$ generate $\mathfrak{A}^{-}$as a von Neumann algebra. If $F$ is a minimal projection in (the type I factor) $\mathfrak{A}(M)^{-}$and $A$ is in $\mathfrak{A}(M)$, then $F A F=a F$ for some scalar $a$. Thus, with $B$ in $\mathscr{N}, F A B F=F A F B=a F B$. Since finite linear combinations of products $A B$ are strong-operator dense in $\mathfrak{A}^{-}, F \mathfrak{A}^{-} F$ is generated as a von Neumann algebra by $F \mathscr{N} F(=\mathscr{N} F)$ and $F \mathfrak{A}(M)^{-} F(=\{a F\})$. Thus $F \mathfrak{A}^{-} F=\mathscr{N} F$. If $T$ is in $\mathfrak{A}(M)^{\prime} \wedge \mathfrak{A}^{-}$, $T F=B F$, for some $B$ in $\mathscr{N}$. Since $A^{\prime} \rightarrow A^{\prime} F$ is an isomorphism of $\mathfrak{A}(M)^{\prime}$ onto $\mathfrak{A}(M)^{\prime} F ; T=B$. Hence $\mathscr{N}=\mathfrak{A}(M)^{\prime} \wedge \mathfrak{A}^{-}$.

3.4. Lemma. If $\mathfrak{A}$ is the CAR algebra based on $\mathscr{H}, \mathfrak{A}$ acts on $\mathscr{H}_{0}$, $M_{1} \subseteq M_{2} \subseteq \cdots \subseteq M_{n}$ are subspaces of $\mathscr{H}$ such that $\mathfrak{A}\left(M_{j}\right)^{-}$is a type I factor and $\varrho$ is an even vector state $\omega_{x} \mid \mathfrak{U}^{-}$of $\mathfrak{Q}^{-}$, then the product $\varrho^{\times}$ relative to the orthogonal decomposition $\left\{N_{1}, \ldots, N_{n}, \mathscr{H} \ominus M_{n}\right\}$ of $\mathscr{H}$, where $N_{j}=M_{j} \ominus M_{j-1}\left(M_{0}=0\right)$, coincides with the restriction $\varrho^{\otimes}$ to $\mathfrak{A}$ of the product of the normal states $\omega_{x}\left|\mathfrak{A}\left(M_{n}\right)^{\prime}, \varrho\right| \mathscr{N}_{1}, \ldots, \varrho \mid \mathscr{N}_{n}$, where $\mathscr{N}_{1}$ $=\mathfrak{A}\left(M_{1}\right)^{-}$and $\mathscr{N}_{j}=\mathfrak{A}\left(M_{j-1}\right)^{\prime} \wedge \mathfrak{U}\left(M_{j}\right)^{-}, j=2, \ldots, n$.

Proof. Of course $\varrho^{\times}$and $\varrho^{\otimes}$ agree on $\mathfrak{A}\left(M_{1}\right)$. Suppose they agree on $\mathfrak{A}\left(M_{j}\right)$. From Lemma $3.3, \mathscr{N}_{j+1}$ is generated linearly by products $a^{\prime}\left(g_{1}\right) \ldots a^{\prime}\left(g_{2 m}\right)$ and $U_{j} a^{\prime}\left(g_{1}\right) \ldots a^{\prime}\left(g_{2 m+1}\right)$ with $g_{j}$ in $N_{j+1}$. If $f_{1}, \ldots, f_{n}$ are in $M_{j}$,

$$
\begin{aligned}
\varrho^{\times}\left(a^{\prime}\left(f_{1}\right) \ldots a^{\prime}\left(f_{n}\right) a^{\prime}\right. & \left.\left(g_{1}\right) \ldots a^{\prime}\left(g_{2 m}\right)\right) \\
& =\varrho^{\times}\left(a^{\prime}\left(f_{1}\right) \ldots a^{\prime}\left(f_{n}\right)\right) \varrho\left(a^{\prime}\left(g_{1}\right) \ldots a^{\prime}\left(g_{2 m}\right)\right) \\
& =\varrho^{\otimes}\left(a^{\prime}\left(f_{1}\right) \ldots a^{\prime}\left(f_{n}\right) a^{\prime}\left(g_{1}\right) \ldots a^{\prime}\left(g_{2 m}\right)\right),
\end{aligned}
$$

and

$$
\begin{aligned}
& \varrho^{\times}\left(a^{\prime}\left(f_{1}\right) \ldots a^{\prime}\left(f_{n}\right) a^{\prime}\left(g_{1}\right) \ldots a^{\prime}\left(g_{2 m+1}\right)\right) \\
& \quad=\varrho^{\times}\left(a^{\prime}\left(f_{1}\right) \ldots a^{\prime}\left(f_{n}\right)\right) \varrho\left(a^{\prime}\left(g_{1}\right) \ldots a^{\prime}\left(g_{2 m+1}\right)\right) \\
& =0 .
\end{aligned}
$$


Now $U_{j}$ is a strong-operator limit of even elements, so that $U_{j} a^{\prime}\left(g_{1}\right) \ldots a^{\prime}\left(g_{2 m+1}\right)$ is a strong-operator limit of odd elements. Thus

$$
\begin{aligned}
& \omega_{x}\left(U_{j} a^{\prime}\left(g_{1}\right) \ldots a^{\prime}\left(g_{2 m+1}\right)\right) \\
&=0=\varrho^{\otimes}\left(a^{\prime}\left(f_{1}\right) \ldots a^{\prime}\left(f_{n}\right) U_{j}\right) \varrho\left(U_{j} a^{\prime}\left(g_{1}\right) \ldots a^{\prime}\left(g_{2 m+1}\right)\right) \\
&=\varrho^{\otimes}\left(a^{\prime}\left(f_{1}\right) \ldots a^{\prime}\left(f_{n}\right) a^{\prime}\left(g_{1}\right) \ldots a^{\prime}\left(g_{2 m+1}\right)\right) .
\end{aligned}
$$

Thus $\varrho^{\otimes}$ and $\varrho^{\times}$agree on $\mathfrak{A}\left(M_{n}\right)$. The argument just given applies, with $\mathscr{H} \ominus M_{n}$ in place of $N_{j+1}$, to show, now, that $\varrho^{\otimes}$ and $\varrho^{\times}$coincide on $\mathfrak{A}$.

3.5. Lemma. If $\left\{\mathscr{U}_{n}\right\}(=\mathscr{P})$ is a family of mutually disjoint bounded regions in $\mathbb{R}^{3}$ with union $\mathscr{U}, M_{n}$ is the subspace of $L_{2}\left(\mathbb{R}^{3}\right)(=\mathscr{H})$ corresponding to $\mathscr{U}_{1} \vee \cdots \vee \mathscr{U}_{n}, M$ corresponds to $\mathscr{U}, \varrho$ is an even locally Fock state of the $C A R$ algebra $\mathfrak{U}$ based on $\mathscr{H}, \mathfrak{U}$ acting on $\mathscr{H}_{0}$ is the representation engendered by $\varrho$ and $\varrho=\omega_{x} \mid \mathfrak{H}, \varrho_{n}^{\otimes}$ is the product of $\omega_{x} \mid \mathfrak{H}\left(M_{n}\right)^{\prime}$, $\omega_{x} \mid\left(\mathfrak{U}\left(M_{j}\right)^{\prime} \wedge \mathfrak{U}\left(M_{j+1}\right)^{-}\right), j=0, \ldots, n-1$, and $\mathfrak{X}_{n}$ is the $C^{*}$-subalgebra of $\mathfrak{N}$ generated by $\mathfrak{A}\left(M_{n}\right)$ and $\mathfrak{U}(\mathscr{H} \ominus M)$; then $\left(\tilde{\varrho}_{n}^{\otimes}\right)$ is $w^{*}$-convergent on $\tilde{\mathfrak{U}}$, the norm closure of $\bigvee_{n} \mathfrak{U}_{n}^{-}$, to a state $\tilde{\varrho}_{\mathscr{P}}^{\otimes}$, and $\varrho_{\mathscr{P}}^{\otimes}\left(=\tilde{\varrho}_{\mathscr{P}}^{\otimes} \mid \mathfrak{Q}\right)$ coincides with $\varrho_{\mathscr{P}}^{\times}$, the Powers factorization of $\varrho$ relative to the orthogonal decomposition $\left(\mathscr{H} \ominus M, N_{1}, N_{2}, \ldots\right\}$ of $\mathscr{H}$, where $N_{j}=M_{j} \ominus M_{j-1}$.

Proof. With $\varrho_{n}^{\times}$the Powers factorization of $\varrho$ relative to $N_{1}, \ldots, N_{n}$, $\mathscr{H} \ominus M_{n}$, we have $\varrho_{n}^{\otimes}=\varrho_{n}^{\times}$, from Lemma 3.4, where $\varrho_{n}^{\otimes}=\varrho_{n}^{\otimes} \mid \mathfrak{A}$. Now $\varrho_{\mathscr{P}}^{\times}$and $\varrho_{n}^{\times}$agree on $\mathfrak{U}_{n}$. Thus $\varrho_{m}^{\otimes} \mid \mathfrak{A}_{m}$ is an extension of $\varrho_{n}^{\otimes} \mid \mathfrak{A}_{n}$, when $n<m$. From Lemma 3.1, $\tilde{\varrho}_{n}^{\otimes}$ is normal; and, as it is the unique normal extension of $\varrho_{n}^{\otimes}$ from $\mathfrak{H}$ to $\mathfrak{H}^{-}, \tilde{\varrho}_{m}^{\otimes} \mid \mathfrak{H}_{m}^{-}$is an extension of $\tilde{\varrho}_{n}^{\otimes} \mid \mathfrak{H}_{n}^{-}$, when $n<m$. Thus $\left(\tilde{\varrho}_{n}^{\otimes} \mid \tilde{\mathfrak{I}}\right)$ is $w^{*}$-convergent to a state $\tilde{\varrho}_{\mathscr{P}}^{\otimes}$ on $\tilde{\mathfrak{A}}$. Since $\tilde{\varrho}_{\mathscr{P}}^{\otimes} \mid \mathfrak{P}_{n}$ $=\varrho_{n}^{\otimes}\left|\mathfrak{U}_{n}=\varrho_{n}^{\times}\right| \mathfrak{U}_{n}=\varrho_{\mathscr{P}}^{\times} \mid \mathfrak{U}_{n}$ and $\bigvee_{n} \mathfrak{U}_{n}$ is norm dense in $\mathfrak{U} ; \varrho_{\mathscr{P}}^{\otimes}=\varrho_{\mathscr{P}}^{\otimes} \mid \mathfrak{U}$ $=\varrho_{\mathscr{P}}^{\times}$.

3.6. Proposition. If $\varphi$ is a quasi-free state of the CAR algebra $\mathfrak{A}$ based on $\mathscr{H}$ and $\left\{M_{a}\right\}$ is an orthogonal decomposition of $\mathscr{H}$, then $\varphi^{\times}$, the factorization of $\varphi$ relative to $\left\{M_{a}\right\}$, is a quasi-free state.

Proof. The value of the truncated $n$-point function of $\varphi^{\times}$is a sum of the values of the truncated $n$-point function of $\varphi$ restricted to the subspaces $M_{a}$, from the definition of $\varphi^{\times}$and [5; Lemma 5.16]. Thus the truncated $n$-point functions of $\varphi^{\times}$vanish for $n>2$; and $\varphi^{\times}$is quasi-free.

\section{§ 4. A Space-factoring Property of Primary Locally Normal States}

A primary, locally normal state $\varrho$ of the CAR algebra $\mathfrak{A}$ enjoys a "clustering property". A slight generalization [3; Proposition 13] to type I funnels of a result [6; Theorem 2.5] of Powers states that: if $\mathcal{O}_{0}$ 
is a bounded region of $\mathbb{R}^{3}, \varepsilon>0$, and $A$ is in the unit ball of $\mathfrak{A}\left(\mathcal{O}_{0}\right)$, the CAR subalgebra of $\mathfrak{A}$ corresponding to $\mathcal{O}_{0}$, then there is a bounded region $\mathcal{O}$ of $\mathbb{R}^{3}$ containing $\mathcal{O}_{0}$ such that $|\varrho(A B)-\varrho(A) \varrho(B)|<\varepsilon$ for each $B$ in the unit ball of $\mathfrak{A}\left(\mathcal{O}^{\prime}\right)$, where $\mathcal{O}^{\prime}$ is the complement of $\mathcal{O}$ in $\mathbb{R}^{3}$. A stronger result, in the direction of space-factorization, would state: $\left\|\varrho \mid \mathfrak{A}_{0}-\varrho_{0}^{\times}\right\|$ $<\varepsilon$, where $\mathfrak{A}_{0}$ is $\mathfrak{A}\left(\mathcal{O}_{0} \cup \mathcal{O}^{\prime}\right)$ and $\varrho_{0}^{\times}$is the product of the restrictions of $\varrho$ to $\mathfrak{A}\left(\mathcal{O}_{0}\right)$ and to $\mathfrak{A}\left(\mathcal{O}^{\prime}\right)$. (We need no longer distinguish between the Powers product $\varrho_{0}^{\times}$and our "commutative" product $\varrho_{0}^{\otimes}$ ). This result is proved in the proposition which follows. (It contains Derek Robinson's extension of the clustering property [7; Theorem 7] in which $A$ is not preassigned; but not Erling Størmer's result [8; Theorem] for the case of ITPFI factors.)

4.1. Proposition. If $\mathfrak{A}$ is a $C^{*}$-algebra acting on the Hilbert space $\mathscr{H}$, $x$ is a cyclic unit vector for $\mathfrak{A}$, and $\left\{\mathscr{M}_{a}\right\}$ is a funnel of type $\mathrm{I}$ factors on $\mathscr{H}$ with union norm-dense in $\mathfrak{A}$; then $\mathfrak{A}^{-}$is a factor if and only if $\left\|\omega_{x}\left|\tilde{\mathfrak{A}}_{a}-\omega_{x}^{\times}\right| \tilde{\mathfrak{A}}_{a}\right\| \rightarrow{ }_{a} 0$, for each $a_{0}$, where $\tilde{\mathfrak{A}}_{a}$ is the von Neumann algebra generated by $\mathscr{M}_{a_{0}}$ and $\mathscr{M}_{a}^{\prime} \cap \mathfrak{H}^{-}$and $\omega_{x}^{\times}$is the factorization of $\omega_{x} \mid \mathfrak{H}^{-}$ relative to $\mathscr{M}_{a_{0}}$.

We prove this proposition with the aid of the following lemmas.

4.2. Lemma. If $\mathscr{M}$ is a type I factor acting on a Hilbert space $\mathscr{K}$ with commutant $\mathscr{M}^{\prime}$ (of type I), $\mathscr{R}$ is a von Neumann subalgebra of $\mathscr{M}$, and $\left(\mathscr{R}_{a}\right)$ is a decreasing net of von Neumann algebras in $\mathscr{M}^{\prime}$ with intersection $\mathscr{R}_{0}$, then

$$
\wedge_{a}\left\{\mathscr{R}, \mathscr{R}_{a}\right\}^{\prime \prime}=\left\{\mathscr{R}, \mathscr{R}_{0}\right\}^{\prime \prime}
$$

Proof. Viewing $\mathfrak{B}(\mathscr{K})$ as $\mathfrak{B}(\mathscr{H}) \otimes \mathfrak{B}\left(\mathscr{H}^{\prime}\right)$ with $\mathscr{M}$ as $\mathfrak{B}(\mathscr{H}) \otimes I$ and $\mathscr{M}^{\prime}$ as $I \otimes \mathfrak{B}\left(\mathscr{H}^{\prime}\right)$; we may view $\mathfrak{B}(\mathscr{H}) \otimes \mathscr{R}_{a}$ as the set of those $m \times m$ matrices with entries in $\mathscr{R}_{a}$ which yield bounded operators when acting on the $m$-fold direct sum of $\mathscr{H}^{\prime}$ with itself. Thus

$$
\mathscr{R} \otimes \mathscr{R}_{0} \cong \wedge_{a}\left(\mathscr{R} \otimes \mathscr{R}_{a}\right)(=\mathscr{J}) \subseteq \mathfrak{B}(\mathscr{H}) \otimes \mathscr{R}_{0}=\wedge_{a}\left(\mathfrak{B}(\mathscr{H}) \otimes \mathscr{R}_{a}\right) .
$$

Now, $\mathscr{R} \otimes \mathscr{R}_{a}$ commutes with $\mathscr{R}^{\prime} \otimes I$; and $\mathfrak{B}(\mathscr{H}) \otimes \mathscr{R}_{0}$ commutes with $I \otimes \mathscr{R}_{0}^{\prime}$. Thus $\mathscr{J}$ commutes with both, and, hence, $\mathscr{J}$ commutes with $\mathscr{R}^{\prime} \otimes \mathscr{R}_{0}^{\prime}$. It follows that $\mathscr{J} \cong\left(\mathscr{R}^{\prime} \otimes \mathscr{R}_{0}^{\prime}\right)^{\prime}=\mathscr{R} \otimes \mathscr{R}_{0} \quad$ [9; Theorem 12.3]; so that

$$
\mathscr{J}=\bigwedge_{a}\left(\mathscr{R} \otimes \mathscr{R}_{a}\right)=\mathscr{R} \otimes \mathscr{R}_{0} .
$$

4.3. Lemma. If $\left\{\mathscr{M}_{a}\right\}$ is a directed family of von Neumann algebras acting on $\mathscr{H}, \mathscr{M}_{a} \subseteq \mathscr{M}_{a^{\prime}}$ if $a^{\prime} \leqq a$, and $\omega$ is a normal functional on $\left\{\underset{a}{\bigvee} \mathscr{M}_{a}\right\}^{\prime \prime}$ 
such that $\omega \mid\left(\wedge_{a} \mathscr{M}_{a}\right)=0$, then $\lim _{a}\left\|\omega \mid \mathscr{M}_{a}\right\|=0$. In particular, if $\wedge_{a}^{\wedge} \mathscr{M}_{a}$ $=\{\lambda I\}$ and $\omega_{1}, \omega_{2}$ are normal states of $\left\{\underset{a}{\bigvee} \mathscr{M}_{a}\right\} " \lim _{a}\left\|\left(\omega_{1}-\omega_{2}\right) \mid \mathscr{M}_{a}\right\|=0$.

Proof. Since the unit ball of each $\mathscr{M}_{a}$ is weak-operator compact and $\omega$ is weak-operator continuous on it, there is an operator $A_{a}$ in it such that $\omega\left(A_{a}\right)=\left\|\omega \mid \mathscr{M}_{a}\right\|$. Now $\left\{A_{a}\right\}$ has a subnet $\left\{A_{a^{\prime}}\right\}$ weak-operator convergent to some $A$. Since $\left\{\mathscr{M}_{a}\right\}$ is directed inversely by inclusion, $A \in \bigwedge_{a} \mathscr{M}_{a}$. Thus $0=\omega(A)=\lim _{a^{\prime}} \omega\left(A_{a^{\prime}}\right)=\lim _{a^{\prime}}\left\|\omega \mid \mathscr{M}_{a^{\prime}}\right\|$. As $\left\{\mathscr{M}_{a^{\prime}}\right\}$ is cofinal in $\left\{\mathscr{M}_{a}\right\}$ and $\left\|\omega\left|\mathscr{M}_{a}\|\leqq\| \omega\right| \mathscr{M}_{a^{\prime}}\right\|$ if $a^{\prime} \leqq a, \lim _{a}\left\|\omega \mid \mathscr{M}_{a}\right\|=0$.

Proof of Proposition 4.1. If $\mathscr{C}$ is the center of $\mathfrak{Q}^{-}$, then

$$
\mathscr{C}=\wedge_{a}\left(\mathscr{M}_{a}^{\prime} \wedge \mathfrak{Q}^{-}\right)
$$

for clearly, $\mathscr{C}$ is contained in this intersection, while the reverse inclusion follows, at once, from the fact that $\mathfrak{A}^{-}$is generated by $\left\{\mathscr{M}_{a}\right\}$. From Lemma 4.2, $\underset{a}{\wedge} \tilde{\mathfrak{A}}_{a}=\left\{\mathscr{M}_{a_{0}}, \mathscr{C}\right\}^{\prime \prime}$. If $\mathfrak{A}^{-}$is a factor, $\mathscr{C}$ is the algebra of scalars, and $\wedge \tilde{\mathfrak{A}}_{a}=\mathscr{M}_{a_{0}}$. Since $\omega_{x} \mid \mathfrak{A}^{-}$and $\omega_{x}^{\times}$agree on $\mathscr{M}_{a_{0}}$, Lemma 4.3 applies; and $\left\|\omega_{x}\left|\tilde{\mathfrak{A}}_{a}-\omega_{x}^{\times}\right| \tilde{\mathfrak{A}}_{a}\right\| \rightarrow_{a} 0$.

Assume, now, that $\left\|\omega_{x}\left|\mathfrak{A}_{a}-\omega_{x}^{\times}\right| \tilde{\mathfrak{A}}_{a}\right\| \rightarrow \vec{a} 0$. If $C \in \mathscr{C}$ and $A_{0} \in \mathscr{M}_{a_{0}}$, $C \in \wedge_{a}\left(\mathscr{M}_{a}^{\prime} \wedge \mathfrak{U}^{-}\right)$; so that $\omega_{x}\left(A_{0} C\right)=\omega_{x}\left(A_{0}\right) \omega_{x}(C)$. As $\underset{a}{\vee} \mathscr{M}_{a}$ is weakoperator dense in $\mathfrak{A}^{-}, \omega_{x}(A C)=\omega_{x}(A) \omega_{x}(C)$, for all $A$ in $\mathfrak{A}^{-}$and $C$ in $\mathscr{C}$. As $x$ is cyclic for $\mathfrak{A}^{-}$, it is separating for $\mathscr{C}$. Thus $\mathscr{C}=\{\lambda I\}$.

4.4. Lemma. If $\mathfrak{A}$ acting on $\mathscr{H}$ is a representation of the CAR algebra, $x$ is a cyclic unit vector for $\mathfrak{A}, \omega_{x} \mid \mathfrak{A}$ is a primary, even, locally Fock state of $\mathfrak{U},\left\{M_{a}\right\}$ is a net of subspaces of $L_{2}\left(\mathbb{R}^{3}\right)$ directed by inclusion, $\underset{a}{\vee} M_{a}$ is dense in $L_{2}\left(\mathbb{R}^{3}\right)$, each $M_{a}$ corresponds to a bounded region of $\mathbb{R}^{3}, M_{a}^{\prime}$ is the complement of $M_{a}$ in $L_{2}\left(\mathbb{R}^{3}\right), \tilde{\mathfrak{A}}_{a}=\mathfrak{A}\left(M_{a_{0}}+M_{a}^{\prime}\right)^{-}$, and $\tilde{\mathfrak{A}}_{0}=\underset{a>a_{0}}{\wedge} \tilde{\mathfrak{A}}_{a}$, then $\tilde{\mathfrak{A}}_{0}=\mathfrak{A}\left(M_{a_{0}}\right)^{-}$.

Proof. From the hypothesis that $x$ is cyclic for $\mathfrak{A}$ and $\omega_{x} \mid \mathfrak{A}$ is even, the main automorphism of $\mathfrak{A}$ [determined by the mapping $\left.a^{\prime}(f) \rightarrow a^{\prime}(-f)\right]$ is implemented by a unitary operator on $\mathscr{H}$ and extends to an automorphism $\alpha$ of $\mathfrak{A}^{-}$. Since $\omega_{x} \mid \mathfrak{A}$ is primary, as well, $\mathfrak{A}^{-}$is a factor. Thus $\wedge\left(\mathfrak{I}\left(M_{a}\right)^{\prime} \wedge \mathfrak{U}^{-}\right)$consists of scalars. (We make use of the fact that $\underset{a}{\vee} \mathfrak{U}\left(M_{a}\right)$ is strong-operator dense in $\mathfrak{U}^{-}$for this.) Let $U_{0}$ be the unitary operator in $\mathfrak{A}\left(M_{a_{0}}\right)^{-}$described in Lemma 3.3. Note, for this, that $\mathfrak{A}\left(M_{a_{0}}\right)^{-}$ is a type I factor - from Lemma 3.2 and the fact that $\omega_{x} \mid \mathfrak{A}$ is a locally Fock state. Referring to elements of $\mathfrak{A}^{-}$fixed or transformed to their 
negatives by $\alpha$ as "even" or "odd" elements, respectively, $U_{0}$ is even and commutes with $\mathfrak{A}\left(M_{a_{0}}^{\prime}\right)$. From Lemma 3.3, $\left(\mathfrak{A}_{+}\left(M_{a}^{\prime}\right)+U_{0} \mathfrak{A}_{-}\left(M_{a}^{\prime}\right)\right)^{-}\left(=\overline{\mathfrak{A}}_{a}\right)$ commutes with $\mathfrak{A}\left(M_{a_{0}}\right)^{-}$when $a_{0}<a$, where $\mathfrak{U}_{+}(M)$ and $\mathfrak{U}_{-}(M)$ refer to the subsets of even and odd elements of $\mathfrak{A}(M)$, respectively. In addition $\overline{\mathfrak{A}}_{a}$ is a von Neumann algebra which, together with $\mathfrak{A}\left(M_{a_{0}}\right)^{-}$, generates $\tilde{\mathfrak{A}}_{a}^{a}$ (as a von Neumann algebra). Writing “" $\overline{\mathfrak{A}}_{0}$ " for “ $\wedge \hat{a} \overline{\mathfrak{A}}_{a}^{a_{0}}$ ", Lemma 4.2 applies; and $\wedge \tilde{\mathfrak{A}}_{a}=\left\{\mathfrak{A}\left(M_{a_{0}}\right) \vee \overline{\mathfrak{A}}_{0}\right\}$ ". The proof is complete when we show that $\overline{\mathfrak{A}}_{0}^{a>a_{0}}$ consists of scalars.

If $T$ is an even element of $\overline{\mathfrak{A}}_{0}, T \in \overline{\mathfrak{A}}_{a}$ for each $a>a_{0}$ and $T=\frac{1}{2}(T+\alpha(T))$. Now $\alpha$ is weak-operator continuous on $\mathfrak{A}^{-}$; and the mapping $A \rightarrow \frac{1}{2}(A+\alpha(A))$ maps $\mathfrak{H}_{+}\left(M_{a}^{\prime}\right)+U_{0} \mathfrak{I}_{-}\left(M_{a}^{\prime}\right)$ onto $\mathfrak{U}_{+}\left(M_{a}^{\prime}\right)$. It, therefore, maps $\overline{\mathfrak{A}}_{a}$ onto $\mathfrak{A}_{+}\left(M_{a}^{\prime}\right)^{-}$. Thus $T \in \mathfrak{A}_{+}\left(M_{a}^{\prime}\right)^{-}\left(\cong \mathfrak{A}\left(M_{a}\right)^{\prime} \wedge \mathfrak{A}^{-}\right)$ for each $a \geqq a_{0}$. It follows that $T$ lies in the center of $\mathfrak{A}^{-}$and is a scalar.

If $T$ is odd, similar use of the mapping $A \rightarrow \frac{1}{2}(A-\alpha(A))$ yields that $T \in U_{0} \mathfrak{A}_{-}\left(M_{a}^{\prime}\right)^{-}$for each $a \geqq a_{0}$. Thus $U_{0} T \in \mathfrak{A}_{-}\left(M_{a}^{\prime}\right)^{-}$. We complete the proof by showing that $\wedge \mathfrak{A}_{-}\left(M_{a}^{\prime}\right)^{-}=(0)$. If $A$ is a self-adjoint operator in this intersection, $A$ is odd. The set of elements in $\mathfrak{A}_{-}\left(M_{a}^{\prime}\right)^{-}$ with which $A$ anticommutes is weak-operator closed in $\mathfrak{A}_{-}\left(M_{a}^{\prime}\right)^{-}$. On the other hand, $A$ anticommutes with $\mathfrak{A}_{-}(M)$, where $M$ is a subspace of $M_{a}^{\prime}$ corresponding to a bounded region of $\mathbb{R}^{3}$. As $\vee \mathfrak{A}_{-}(M)$ is weakoperator dense in $\mathfrak{A}_{-}\left(M_{a}^{\prime}\right)^{-}, A$ anticommutes with $\mathfrak{A}_{-}\left(M_{a}^{\prime}\right)^{-}$and, in particular, with itself. Thus $A=0$.

4.5. Proposition. If $\mathfrak{A}$ is the CAR algebra over $\mathscr{H}\left(=L_{2}\left(\mathbb{R}^{3}\right)\right),\left\{M_{a}\right\}$ is a net of subspaces of $\mathscr{H}$, directed by inclusion, corresponding to bounded regions with union $\mathbb{R}^{3}, \varrho$ is an even, locally Fock state of $\mathfrak{A}, \mathfrak{A}_{a}$ is the $C^{*-}$ subalgebra of $\mathfrak{A}$ generated by $\mathfrak{I}\left(M_{a_{0}}\right)$ and $\mathfrak{A}\left(\mathscr{H} \Theta M_{a}\right)$, and $\varrho_{a_{0}}^{\times}$is the factorization of $\varrho$ relative to $\mathfrak{A}\left(M_{a_{0}}\right)$; then $\left\|\varrho\left|\mathfrak{A}_{a}-\varrho_{a_{0}}^{\times}\right| \mathfrak{A}_{a}\right\| \rightarrow \vec{a} 0$, for each $a_{0}$, if and only if $\varrho$ is primary.

Proof. Passing to the representation of $\mathfrak{A}$ obtained by applying the GNS construction to $\varrho$, we may assume that $\mathscr{A}$ acts on $\mathscr{H}_{0}, \varrho$ is an even, vector state $\omega_{x} \mid \mathfrak{A}$ of $\mathfrak{A}, x$ is a cyclic unit vector for $\mathfrak{A}$, and $\mathfrak{A}\left(M_{a}\right)^{-}$is a type I factor. Applying Lemma 3.4, $\varrho_{a_{0}}^{\times}=\varrho_{a_{0}}^{\otimes}$, and $\varrho_{a_{0}}^{\times}$is the restriction of a normal state $\tilde{\varrho}_{a_{0}}^{\times}$of $\mathfrak{A}^{-}$to $\mathfrak{A}$. Now $\tilde{\varrho}_{a_{0}}^{\times}\left|\mathfrak{A}\left(M_{a_{0}}\right)^{-}=\omega_{x}\right| \mathfrak{A}\left(M_{a_{0}}\right)^{-}$; and $\underset{a>a_{0}}{\wedge} \mathfrak{I}_{a}^{-}=\mathfrak{A}\left(M_{a_{0}}\right)^{-}$, if $\varrho$ is primary (from Lemma 4.4). Lemma 4.3 applies, in this case, and

$$
\left\|\varrho\left|\mathfrak{A}_{a}-\varrho_{a_{0}}^{\times}\right| \mathfrak{A}_{a}\right\|=\left\|\omega_{x}\left|\mathfrak{A}_{a}^{-}-\tilde{\varrho}_{a_{0}}^{\times}\right| \mathfrak{H}_{a}^{-}\right\| \vec{a}_{a} 0 .
$$

If the preceding limit is valid for each $a_{0}$, Proposition 4.1 applies, since $\left\{\mathfrak{U}\left(M_{a}\right)^{-}\right\}$is a funnel of type I factors for $\mathfrak{I}^{-}$and $\mathfrak{A}_{a}^{-}$is the von Neumann algebra generated by $\mathfrak{U}\left(M_{a_{0}}\right)^{-}$and $\mathfrak{A}\left(M_{a}\right)^{\prime} \wedge \mathfrak{U}^{-}$. Thus $\varrho$ is primary. 


\section{§ 5. States With No Long-Range Correlations}

Proposition 4.5 assures us that $\left\|\left(\varrho-\varrho^{\times}\right) \mid \mathfrak{A}\left(\mathcal{O}_{0} \vee \mathcal{O}\right)\right\|$ tends to 0 , for a fixed bounded region $\mathcal{O}_{0}$ of $\mathbb{R}^{3}$, as the distance $d\left(\mathcal{O}_{0}, \mathcal{O}\right)$ between $\mathcal{O}_{0}$ and $\mathcal{O}$ tends to $\infty$, where $\varrho$ is an even, primary, locally Fock state of the CAR algebra $\mathfrak{A}$ and $\varrho^{\times}$is its factorization relative to $\mathfrak{U}\left(\mathcal{O}_{0}\right)$. This norm will depend in general not only on the separation between $\mathcal{O}_{0}$ and $\mathcal{O}$ but also on the shape and location of $\mathcal{O}_{0}$. It appears, however, reasonable, from the physical point of view, to limit attention to the class of states for which the dependence on $\mathcal{O}_{0}$ may be eliminated. We introduce a function $\delta_{\varrho}(d)$, called the modulus of decoupling for the state $\varrho$, defined by

$$
\begin{array}{r}
\delta_{\varrho}\left(d_{0}\right)=\sup \left\{\left\|\left(\varrho-\varrho^{\times}\right) \mid \mathfrak{H}\left(\mathcal{O}_{1} \vee \mathcal{O}_{2}\right)\right\|: \mathcal{O}_{1}, \mathcal{O}_{2} \text { bounded regions of } \mathbb{R}^{3},\right. \\
\left.d\left(\mathcal{O}_{1}, \mathcal{O}_{2}\right) \geqq d_{0}\right\}
\end{array}
$$

where $\varrho^{\times}$is the factorization relative to $\mathfrak{A}\left(\mathcal{O}_{1}\right)$. Note that $\delta_{\varrho}$ is bounded by 2 .

5.1. Definition. If $\delta_{\varrho}(d) \rightarrow 0$ as $d \rightarrow \infty$, we say that $\varrho$ has no long-range correlations.

This suggests the study of states of $\mathfrak{U}$ for which $\delta_{\varrho}$ has a prescribed rate of decay $($ at $\infty)$. The discussion which follows leads to Theorem 5.5 which is concerned with states $\varrho$ for which $\delta_{\varrho}(d)=\mathcal{O}\left([\log d]^{-(1+a)}\right)$ with $a>0$.

5.2. Lemma. If $\varrho$ is an even state of the CAR algebra $\mathfrak{Y}, \varrho$ has no long-range correlations, $\left\{\mathscr{U}_{j}\right\}$ is a set of mutually-disjoint, bounded regions of $\mathbb{R}^{3}, \mathfrak{U}_{j}$ is $\mathfrak{A}\left(\mathscr{U}_{1} \vee \cdots \vee \mathscr{U}_{j}\right), \varrho_{j}^{\times}$is the factorization of $\varrho$ relative to $\left\{\mathscr{U}_{1}, \ldots, \mathscr{U}_{j}\right\}$, and $d_{j+1}$ is the distance from $\mathscr{U}_{j+1}$ to $\mathscr{U}_{1} \vee \cdots \vee \mathscr{U}_{j}$, then

$$
\left\|\left(\varrho-\varrho_{j+1}^{\times}\right)\left|\mathfrak{A}_{j+1}\|\leqq\|\left(\varrho-\varrho_{j}^{\times}\right)\right| \mathfrak{A}_{j}\right\|+\delta_{\varrho}\left(d_{j+1}\right) .
$$

Proof. It will suffice to establish

$$
\left|\left(\varrho-\varrho_{j+1}^{\times}\right)(A)\right| \leqq\left\|\left(\varrho-\varrho_{j}^{\times}\right) \mid \mathfrak{U}_{j}\right\|+\delta_{\varrho}\left(d_{j+1}\right)
$$

for self-adjoint elements $A$ of the form $\sum_{k=1}^{m} A_{1 k} \ldots A_{j k} A_{j+1 k}$ in the unit ball of $\mathfrak{U}_{j+1}$, where $A_{h k} \in \mathfrak{U}\left(\mathscr{U}_{h}\right)$, since $\varrho-\varrho_{j+1}^{\times}$is hermitian and such elements form a norm-dense subset of the self-adjoint elements in the unit ball of $\mathfrak{U}_{j+1}$. If $\tau$ is a state of $\mathfrak{U}_{j}$, from [5; Lemma 5.3], $\tau\left|\mathfrak{U}_{j} \otimes \varrho\right| \mathfrak{U}\left(\mathscr{U}_{j+1}\right)$ is a state of $\mathfrak{U}_{j+1}$. As $-I \leqq \sum_{k=1}^{m} A_{1 k} \ldots A_{j k} A_{j+1 k} \leqq I$, it follows that

$$
-1 \leqq \sum_{k=1}^{m} \tau\left(A_{1 k} \ldots A_{j k}\right) \varrho\left(A_{j+1 k}\right)=\tau\left(\sum_{k=1}^{m} \varrho\left(A_{j+1 k}\right) A_{1 k} \ldots A_{j k}\right) \leqq 1 ;
$$


so that $\sum_{k=1}^{m} \varrho\left(A_{j+1 k}\right) A_{1 k} \ldots A_{j k}$ is self-adjoint and

Thus

$$
\left\|\sum_{k=1}^{m} \varrho\left(A_{j+1 k}\right) A_{1 k} \ldots A_{j k}\right\| \leqq 1 .
$$

$$
\begin{gathered}
\left|\sum_{k=1}^{m} \varrho\left(A_{1 k} \ldots A_{j k}\right) \varrho\left(A_{j+1 k}\right)-\sum_{k=1}^{m} \varrho\left(A_{1 k}\right) \ldots \varrho\left(A_{j k}\right) \varrho\left(A_{j+1 k}\right)\right| \\
\leqq\left\|\left(\varrho-\varrho_{j}^{\times}\right) \mid \mathfrak{A}_{j}\right\| .
\end{gathered}
$$

On the other hand,

$$
\left|\varrho(A)-\sum_{k=1}^{m} \varrho\left(A_{1 k} \ldots A_{j k}\right) \varrho\left(A_{j+1 k}\right)\right| \leqq \delta_{\varrho}\left(d_{j+1}\right) .
$$

Combining $(* *)$ and $(* * *)$, we have $(*)$, completing the proof.

We describe, now, a paving $\mathscr{P}\left(\left(r_{n}\right),\left(d_{n}\right)\right)$ of $\mathbb{R}^{3}$ depending on the choice of two sequences $\left(r_{n}\right)$ and $\left(d_{n}\right)$ of monotonically increasing positive numbers. Lemma 5.3 below describes conditions on $\left(r_{n}\right),\left(d_{n}\right)$ under which $\mathscr{P}\left(\left(r_{n}\right),\left(d_{n}\right)\right)$ is a lacunary paving. Let $s_{n}$ be $\frac{1}{2}\left(r_{n}-r_{n-1}\right)$. We assume that $d_{n}<s_{n}$. The construction proceeds as follows. Let $C_{n}$ be the cube with edge length $r_{n}$, center at the origin and edges parallel to the axes of a Cartesian coordinate system in $\mathbb{R}^{3}$. Let $u_{n}$ be the greatest integer not exceeding $r_{n} / s_{n}$. Place $u_{n}^{2}$ cubes of edge length $s_{n}$ on one face of $C_{n}$ (with edges parallel to the Cartesian frame) and remove the cubes near the four edges of that face. Proceed, a face at a time, placing $u_{n}^{2}$ cubes and removing those along edges not on faces already considered. In this way, we place at least $6 u_{n}^{2}-12 u_{n}\left(=c_{n}\right)$ such cubes in $C_{n} \backslash C_{n-1}$. Now remove cubes until exactly $c_{n}$ remain in $C_{n} \backslash C_{n-1}$. Since $\left(r_{n} / s_{n}\right)-1 \leqq u_{n}$ $\leqq r_{n} / s_{n}$,

$$
6\left(r_{n} / s_{n}\right)^{2}-24\left(r_{n} / s_{n}\right)+6 \leqq c_{n} \leqq 6\left(r_{n} / s_{n}\right)^{2} .
$$

Shrink each of these cubes to one of edge length $s_{n}-d_{n}$ keeping the center fixed and the edges parallel to the coordinate axes. The smaller cubes, in total, constitute the paving $\mathscr{P}\left(\left(r_{n}\right),\left(d_{n}\right)\right)$. Let $\mathscr{U}_{n 1}, \ldots, \mathscr{U}_{n c_{n}}$ be the cubes in $C_{n} \backslash C_{n-1}$.

We estimate the volume in $C_{n} \backslash C_{n-1}$ not covered by $\mathscr{U}_{n 1}, \ldots, \mathscr{U}_{n c_{n}}$. It comes from two sources: the volume not covered by the larger cubes,

$$
\begin{aligned}
\mu\left(C_{n} \backslash C_{n-1}\right)- & -c_{n} s_{n}^{3} \leqq r_{n}^{3}-\left(r_{n}-2 s_{n}\right)^{3} \\
& -s_{n}^{3}\left(6\left(r_{n} / s_{n}\right)^{2}-24\left(r_{n} / s_{n}\right)+6\right) \leqq 12 s_{n}^{2} r_{n}+2 s_{n}^{3},
\end{aligned}
$$

where $\mu$ is Lebesgue measure (volume) in $\mathbb{R}^{3}$, and the gaps introduced by shrinking each of the cubes. Each face of a cube is moved in $\frac{1}{2} d_{n}$ (parallel to itself) in this process, so that the volume loss introduced 
in this way for each cube is less than $6 s_{n}^{2}\left(\frac{1}{2} d_{n}\right)$; and the total volume uncovered in this way is less than $3 c_{n} s_{n}^{2} d_{n} \leqq 18 r_{n}^{2} d_{n}$. In total then, with $\mathscr{U}=\underset{n, j}{\bigvee} \mathscr{U}_{n j}$,

$$
\frac{\mu\left(\mathscr{U}^{\prime} \wedge C_{n}\right)}{\mu\left(C_{n}\right)} \leqq \frac{\sum_{k=1}^{n}\left(12 s_{k}^{2} r_{k}+18 r_{k}^{2} d_{k}+2 s_{k}^{3}\right)}{r_{n}^{3}} .
$$

5.3. Lemma. If $\left(r_{n-1} / r_{n}\right)_{\vec{n}} 1,\left(d_{n} / s_{n}\right){ }_{n} 0$, and $\left(s_{n}\right)$ is monotone increasing, then $\mathscr{P}\left(\left(r_{n}\right),\left(d_{n}\right)\right)$ is a lacunary paving of $\mathbb{R}^{3}$.

Proof. Let $r_{0}$ be 0 . From (2), $\mu\left(\mathscr{U}^{\prime} \wedge C_{n}\right) / \mu\left(C_{n}\right) \leqq \sum_{k=1}^{n} g_{k} / \sum_{k=1}^{n} h_{k}$, where $g_{k}=12 s_{k}^{2} r_{k}+18 r_{k}^{2} d_{k}+2 s_{k}^{3}$ and $h_{k}=s_{k} r_{k-1}^{2} \leqq r_{k}^{3}-r_{k-1}^{3}$. Since $\left(g_{k}\right)$ and $\left(h_{k}\right)$ are monotone increasing and $\left(g_{k} / h_{k}\right) \rightarrow\left(\sum_{k=1}^{n} g_{k} / \sum_{k=1}^{n} h_{k}\right) \rightarrow \vec{n} 0$.

If $B_{n}$ is the ball in $\mathbb{R}^{3}$ with center the origin and radius $\frac{1}{2} r_{n}$, then $\left(a_{n}+b_{n}\right) /\left(1+e_{n}\right)=\mu\left(\mathscr{U} \wedge C_{n}\right) / \mu\left(C_{n}\right)_{\vec{n}} 1$, where $a_{n}=\mu\left(\mathscr{U} \wedge B_{n}\right) / \mu\left(B_{n}\right)$, $b_{n}=\mu\left(\mathscr{U} \wedge\left(C_{n} \backslash B_{n}\right)\right) / \mu\left(B_{n}\right)$, and $e_{n}=\mu\left(C_{n} \backslash B_{n}\right) / \mu\left(B_{n}\right)$. Since $a_{n} \leqq 1$ and $b_{n} \leqq e_{n} \leqq 1 ;\left|1+e_{n}-\left(a_{n}+b_{n}\right)\right|=1-a_{n}+e_{n}-b_{n} \vec{n} 0$; and, thus, $a_{n} \vec{n} 1$.

If $B_{r}$ is a ball in $\mathbb{R}^{3}$ with center the origin and $B_{n-1} \subseteq B_{r} \subseteq B_{n}$, then

$$
\begin{aligned}
\frac{\mu\left(\mathscr{U} \wedge B_{r}\right)}{\mu\left(B_{r}\right)} \geqq \frac{\mu\left(\mathscr{U} \wedge B_{n-1}\right)}{\mu\left(B_{n}\right)} & =\frac{\mu\left(\mathscr{U} \wedge B_{n-1}\right)}{\mu\left(B_{n-1}\right)} \cdot \frac{\mu\left(B_{n-1}\right)}{\mu\left(B_{n}\right)} \\
& =\frac{\mu\left(\mathscr{U} \wedge B_{n-1}\right)}{\mu\left(B_{n-1}\right)} \cdot \frac{r_{n-1}^{3}}{r_{n}^{3}} \vec{n} 1 .
\end{aligned}
$$

Since the diameter of $\mathscr{U}_{n j}$ is not greater than $\sqrt{3} s_{n}$, the outer distance of $\mathscr{U}_{n j}$ is not less than $\frac{1}{2} r_{n-1}$, and $2 s_{n} / r_{n-1} \vec{n} 0$, we conclude that $\mathscr{P}\left(\left(r_{n}\right),\left(d_{n}\right)\right)$ is a lacunary paving.

From the discussion following Definition 2.1, the paving $\mathscr{P}_{n_{0}}\left(\left(r_{n}\right),\left(d_{n}\right)\right)$ obtained from $\mathscr{P}\left(\left(r_{n}\right),\left(d_{n}\right)\right)$ by excising that portion in $C_{n_{0}-1}$ is, again, a lacunary paving.

5.4 Lemma. If $\varrho$ is an even state of the CAR algebra and $\varrho_{n_{0}}^{\times}$is its factorization relative to $\mathscr{P}_{n_{0}}\left(\left(r_{n}\right),\left(d_{n}\right)\right)$, then

$$
\left\|\left(\varrho-\varrho_{n_{0}}^{\times}\right) \mid \mathfrak{A}\left(\mathscr{U}_{n_{0}}\right)\right\| \leqq \sum_{j=n_{0}}^{\infty} c_{j} \delta_{\varrho}\left(d_{j-1}\right) .
$$

Proof. Let $\mathfrak{A}_{n j}$ be $\mathfrak{A}\left(\mathscr{U}_{n_{0} 1} \vee \cdots \vee \mathscr{U}_{n 1} \vee \cdots \vee \mathscr{U}_{n j}\right)$ and $\varrho_{n j}^{\times}$be the factorization of $\varrho$ relative to $\left\{\mathscr{U}_{n_{0} 1}, \ldots, \mathscr{U}_{n j}\right\}$. In the process of shrinking the initial cubes in $C_{n} \backslash C_{n-1}$, the distance between these cubes becomes $d_{n}$; while the distance to cubes in $C_{n-1} \backslash C_{n-2}$ becomes $\frac{1}{2}\left(d_{n-1}+d_{n}\right)$. Since 
$\left(d_{n}\right)$ is monotone increasing, each cube in $C_{n} \backslash C_{n-1}$ is a distance at least $d_{n-1}$ from all other cubes of the paving $\mathscr{P}\left(\left(r_{n}\right),\left(d_{n}\right)\right)$. Thus

$$
\left\|\left(\varrho-\varrho_{n_{0} 2}^{\times}\right) \mid \mathfrak{A}_{n_{0} 2}\right\| \leqq \delta_{\varrho}\left(d_{n_{0}-1}\right) .
$$

Repeated application of Lemma 5.2 yields

$$
\begin{aligned}
\left\|\left(\varrho-\varrho_{n_{0} 3}^{\times}\right) \mid \mathfrak{A}_{n_{0} 3}\right\| & \leqq 2 \delta_{\varrho}\left(d_{n_{0}-1}\right), \ldots, \\
\left\|\left(\varrho-\varrho_{n_{0} c_{n_{0}}}^{\times}\right) \mid \mathfrak{A}_{n_{0} c_{n_{0}}}\right\| & \leqq c_{n_{0}} \delta_{\varrho}\left(d_{n_{0}-1}\right), \ldots, \\
\left\|\left(\varrho-\varrho_{n c_{n}}^{\times}\right) \mid \mathfrak{A}_{n c_{n}}\right\| & \leqq \sum_{j=n_{0}}^{n} c_{j} \delta_{\varrho}\left(d_{j-1}\right) .
\end{aligned}
$$

Since $\underset{n \geqq n_{0}}{\vee} \mathfrak{A}_{n c_{n}}$ is norm-dense in $\mathfrak{A}\left(\mathscr{U}_{n_{0}}\right)$, (3) follows.

For convenient reference, we collect some formulae and limits before beginning the proof of our main theorem.

$$
\frac{d}{d x}\left(e^{x / \log x}\right)=\frac{\log x-1}{(\log x)^{2}} e^{x / \log x} .
$$

It follows from this that

Thus

$$
e^{x / \log x} \text { is increasing for } x \geqq 3 .
$$

and

$$
x / \log x \quad \text { is increasing when } x \geqq 3 \text {, }
$$

$$
\begin{aligned}
0 & \leqq \frac{x}{\log x}-\frac{x-1}{\log (x-1)}=\frac{x \log \frac{x-1}{x}}{(\log x)(\log (x-1))}+\frac{1}{\log (x-1)} \\
& \leqq \frac{1}{\log (x-1)} \rightarrow 0 \text { as } x \rightarrow \infty
\end{aligned}
$$

Hence

$$
\begin{gathered}
\frac{e^{x / \log x}}{e^{(x-1) / \log (x-1)} \rightarrow 1 \quad \text { as } x \rightarrow \infty .} \\
\frac{d}{d x}\left(\frac{e^{x / \log x}}{(\log x)^{2}}\right)=\frac{\left(\log x-1-2 x^{-1} \log x\right)}{(\log x)^{4}} e^{x / \log x} .
\end{gathered}
$$

It follows that

$$
\begin{aligned}
& \frac{e^{x / \log x}}{(\log x)^{2}} \text { is increasing when } x \geqq 8 . \\
& \frac{x}{\log x} \geqq \log \left(\frac{e^{x / \log x}}{(\log x)^{2}}\right)=\frac{x-(2 \log (\log x)) \log x}{\log x} \\
& \geqq \frac{x}{2 \log x} \text { when } x \geqq 30 .
\end{aligned}
$$


Thus

$$
\frac{e^{x / \log x}}{(\log x)^{2}} \rightarrow \infty \quad \text { as } \quad x \rightarrow \infty
$$

5.5. Theorem. If $\varrho$ is an even state of the CAR algebra $\mathfrak{A}, t \rightarrow \alpha_{t}$ is the one-parameter group of time automorphisms of $\mathfrak{U}$ generated by the free Hamiltonian, $\hat{\varrho}$ is the quasi-free state of $\mathfrak{A}$ with the same two-point function as $\varrho, \delta_{\varrho}$ is the modulus of decoupling of $\varrho$, and if $\delta_{\varrho}(d)$ $=\mathcal{O}\left([\log d]^{-(1+a)}\right)$ with a positive, then $\left|(\varrho-\hat{\varrho})\left(\alpha_{t}(A)\right)\right| \rightarrow 0$ as $t \rightarrow \infty$, for each $A$ in $\mathfrak{A}$.

Proof. By hypothesis there are positive constants $d_{0}$ and $k$ such that $\delta_{\varrho}(d) \leqq k(\log d)^{-(1+a)}$ when $d_{0} \leqq d$. Given a positive $\varepsilon$, let $d_{n-1}$ be $e^{n / \log n} /(\log n)^{2}$. Choose $n_{0}(>30)$ such that $d_{n_{0}-1}>d_{0} \quad[\operatorname{see}(8)]$, $\frac{(\log n)^{2}}{\log n-1} \cdot \frac{e^{(n+1) / \log (n+1)}}{e^{n / \log n}}<\sqrt{2} \log n$, when $n \geqq n_{0}[$ see $(5)]$, and

$$
48 k \sum_{n=n_{0}}^{\infty} \frac{(2 \log n)^{3+a}}{\mathrm{n}^{1+a}}<\varepsilon .
$$

Let $r_{n}$ be $r_{n-1}+(\log n-1) d_{n-1}$ when $n \geqq n_{0}$; and let $r_{n_{0}-1}$ be 0 . From (6) and (8), $\left(d_{n}\right)$ is monotone increasing and tends to $\infty$ as $n \rightarrow \infty$. Thus the same is true for $\left(r_{n}\right)$ and $\left(r_{n}-r_{n-1}\right)$. From (5),

$$
\frac{d_{n}}{s_{n}}=2\left(\frac{\log n}{\log (n+1)}\right)^{2} \cdot \frac{e^{(n+1) / \log (n+1)}}{e^{n / \log n}} \cdot \frac{1}{\log n-1} \rightarrow 0 \text {. }
$$

From (4) and (6),

$$
\begin{aligned}
e^{n / \log n}-e^{n_{0} / \log n_{0}} & =\int_{n_{0}}^{n} \frac{\log x-1}{(\log x)^{2}} e^{x / \log x} d x \\
& \leqq \sum_{j=n_{0}}^{n} \frac{\log j-1}{(\log j)^{2}} e^{j / \log j}=r_{n} \leqq \int_{n_{0}}^{n+1} \frac{\log x-1}{(\log x)^{2}} e^{x / \log x} d x \\
& \leqq e^{(n+1) / \log (n+1)} .
\end{aligned}
$$

Thus

$$
1 \leqq \frac{r_{n}}{r_{n-1}} \leqq \frac{e^{(n+1) / \log (n+1)}}{e^{(n-1) / \log (n-1)}-e^{n_{0} / \log n_{0}}} \rightarrow 1
$$

From Lemma 5.3, $\mathscr{P}\left(\left(r_{n}\right),\left(d_{n}\right)\right)$ is a lacunary paving. Since

$$
\delta_{\varrho}\left(d_{n-1}\right) \leqq k\left(\log \left(\frac{e^{n / \log n}}{(\log n)^{2}}\right)\right)^{-(1+a)} \leqq k\left(\frac{n}{2 \log n}\right)^{-(1+a)}
$$


from (7); it follows that

$$
\begin{gathered}
\sum_{n=n_{0}}^{\infty} c_{n} \delta_{Q}\left(d_{n-1}\right) \leqq 6 k \sum_{n=n_{0}}^{\infty}\left(\frac{r_{n}}{s_{n}}\right)^{2}\left(\frac{2 \log n}{n}\right)^{1+a} \\
=24 \mathrm{k} \sum_{n=n_{0}}^{\infty}\left(\frac{r_{n}}{(\log n-1) d_{n-1}}\right)^{2}\left(\frac{2 \log n}{n}\right)^{1+a} \\
\leqq 24 k \sum_{n=n_{0}}^{\infty}\left(\frac{(\log n)^{2}}{\log n-1} \cdot \frac{e^{(n+1) / \log (n+1)}}{e^{n / \log n}}\right)^{2}\left(\frac{2 \log n}{n}\right)^{1+a} \\
\leqq 48 k \sum_{n=n_{0}}^{\infty} \frac{(2 \log n)^{3+a}}{n^{1+a}}<\varepsilon .
\end{gathered}
$$

From Lemma 5.4, $\left\|\left(\varrho-\varrho^{\times}\right) \mid \mathfrak{A}(\mathscr{U})\right\| \leqq \varepsilon$, where $\varrho^{\times}$is the factorization of $\varrho$ relative to $\mathscr{P}\left(\left(r_{n}\right),\left(d_{n}\right)\right)$.

In order to show that $\left|(\varrho-\varrho)\left(\alpha_{t}(A)\right)\right| \rightarrow 0$ as $t \rightarrow \infty$, for each $A$ in $\mathfrak{U}$, it will suffice to prove this for each $A$ in some (norm-) total family (since $\alpha_{t}$ is an isometry of $\mathfrak{A}$ onto $\mathfrak{A})$. We may assume that $A=a^{\prime}\left(f_{1}\right) \ldots a^{\prime}\left(f_{n}\right)$, where $A$ and each $a^{\prime}\left(f_{j}\right)$ have norm 1 . From the definition of a quasifree state, there is a polynomial $p_{n}$ in $\left(\begin{array}{l}n \\ 2\end{array}\right)$ variables such that, for each quasi-free state $\tau$ of $\mathfrak{A}$ and each set $\left\{h_{1}, \ldots, h_{n}\right\}$ of $n$ functions in $L_{2}\left(\mathbb{R}^{3}\right)$, $\tau\left(a^{\prime}\left(h_{1}\right) \ldots a^{\prime}\left(h_{n}\right)\right)=p_{n}\left(\tau\left(a^{\prime}\left(h_{j}\right) a^{\prime}\left(h_{k}\right)\right)\right)(j<k)$. By (uniform) continuity of $p_{n}$ on bounded sets in $\mathbb{R}^{\left(\begin{array}{c}n \\ 2\end{array}\right)}$, given a positive integer $m$, there is a positive $\varepsilon$ $\left(<\frac{1}{m}\right)$ such that $\left|p_{n}\left(a_{j k}\right)-p_{n}\left(a_{j k}^{\prime}\right)\right|<\frac{1}{m}$ provided $\left|a_{j k}\right|+\left|a_{j k}^{\prime}\right| \leqq 4$ and $\left|a_{j k}-a_{j k}^{\prime}\right|<\varepsilon$ for all $j, k(j<k)$. Choose $\mathscr{P}\left(\left(r_{n}\right),\left(d_{n}\right)\right)$, as above, corresponding to this $\varepsilon$ (and to $a$ for the modulus of decoupling of $\varrho$ ). Let $\varrho^{\times}$ and $\hat{\varrho}^{\times}$be the factorizations of $\varrho$ and $\varrho$ relative to $\mathscr{P}\left(\left(r_{n}\right),\left(d_{n}\right)\right)$.

Find $g_{j}$ corresponding to $f_{j}$, as in Lemma 2.2, such that $\left\|g_{j t}-f_{j(t)}\right\|_{2}$ $\rightarrow 0$. Then $\left\|B_{t}-A_{t}\right\| \rightarrow 0$ as $t \rightarrow \infty$, where $B_{t}=a^{\prime}\left(g_{1 t}\right) \ldots a^{\prime}\left(g_{n t}\right)$ and $A_{t}=\alpha_{t}(A)$. There is a positive $t_{0}$ such that $\left|(\varrho-\varrho)\left(A_{t}-B_{t}\right)\right|<\frac{1}{m}$, if $t \geqq t_{0}$. Now $B_{t}$ is a norm-limit of a sum of products of elements of grade two or less - each vanishing outside some $\mathscr{U}_{n j}$. Hence $\varrho^{\times}\left(B_{t}\right)=\varrho^{\times}\left(B_{t}\right)$. From Proposition 3.6, $\varrho^{\times}$is quasi-free, so that

Since

$$
\varrho^{\times}\left(B_{t}\right)=p_{n}\left(\varrho^{\times}\left(a^{\prime}\left(g_{j t}\right) a^{\prime}\left(g_{k t}\right)\right)\right) \text {. }
$$

and

$$
\left|\left(\varrho-\varrho^{\times}\right)\left(a^{\prime}\left(g_{j t}\right) a^{\prime}\left(g_{k t}\right)\right)\right|<\varepsilon
$$

$$
\left|\varrho^{\times}\left(a^{\prime}\left(g_{j t}\right) a^{\prime}\left(g_{k t}\right)\right)\right|+\left|\varrho\left(a^{\prime}\left(g_{j t}\right) a^{\prime}\left(g_{k t}\right)\right)\right| \leqq 4 ;
$$


we have

$$
\begin{aligned}
\left|(\varrho-\hat{\varrho})\left(B_{t}\right)\right| & \leqq\left|\left(\varrho-\varrho^{\times}\right)\left(B_{t}\right)\right|+\left|\left(\hat{\varrho}^{\times}-\hat{\varrho}\right)\left(B_{t}\right)\right| \\
& \leqq \varepsilon+\left|p_{n}\left(\varrho^{\times}\left(a^{\prime}\left(g_{j t}\right) a^{\prime}\left(g_{k t}\right)\right)\right)-p_{n}\left(\varrho\left(a^{\prime}\left(g_{j t}\right) a^{\prime}\left(g_{k t}\right)\right)\right)\right| \\
& =\varepsilon+\left|p_{n}\left(\varrho^{\times}\left(a^{\prime}\left(g_{j t}\right) a^{\prime}\left(g_{k t}\right)\right)\right)-p_{n}\left(\varrho\left(a^{\prime}\left(g_{j t}\right) a^{\prime}\left(g_{k t}\right)\right)\right)\right| \\
& <\frac{2}{m} .
\end{aligned}
$$

Thus $\left|(\varrho-\varrho)\left(A_{t}\right)\right|<\frac{3}{m}$, if $t \geqq t_{0}$ and $\left|(\varrho-\hat{\varrho})\left(A_{t}\right)\right| \rightarrow 0$ as $t \rightarrow \infty$.

Acknowledgement. The authors are grateful to the National Science Foundation (USA), the Ministère de l'Education Nationale (France), and the Guggenheim Foundation, for the support they provided at various stages in the development of this work.

\section{References}

1. Haag, R.: Asymptotic behavior in time of the states of a free Fermi gas. Colloques internationaux du CNRS, $\mathrm{N}^{\circ} 181$, Paris 1970 p. $143-145$

2. Haag, R.: Quantum statistical mechanics of infinite systems. In "Problems of Theoretical Physics", N.N.Bogolubov birthday volume, Nauka, Moscow 1969, p. 379-385

3. Haag, R., Kadison, R., Kastler, D.: Nets of $C^{*}$-algebras and classification of states. Commun. Math. Phys. 16, 81-104 (1970)

4. Lanford,O., Robinson, D.: Approach to equilibrium of free quantum systems. Commun. Math. Phys. 24, 193-210 (1972)

5. Powers, R.: Representations of the canonical anticommutation relations. Thesis Princeton Univ. (1967)

6. Powers, R.: Representations of uniformly hyperfinite algebras and their associated rings. Ann. Math. 86, 138-171 (1967)

7. Robinson, D.: Normal and locally normal states. Commun. Math. Phys. 19, 219-234 (1970)

8. Størmer,E.: Hyperfinite product factors. Arkiv för matematik 9, 165-170 (1971)

9. Takesaki,M.: Tomita's theory of modular Hilbert algebras and its applications. Berlin-Heidelberg-New York: Springer 1970

R. V. Kadison

Department of Mathematics

University of Pennsylvania

Philadelphia, Pa. 19174, USA

D. Kastler

CPT, CNRS

31 Chemin Joseph Aiguier

F-13 Marseille $9^{\mathrm{e}}$, France

\section{R. Haag}

Institut für Theoretische Physik

der Universität

D-2000 Hamburg 50

Luruper Chaussee 149

Federal Republic of Germany 